\title{
Topological Lensing in Spherical Spaces
}

\author{
Evelise Gausmann ${ }^{1}$, Roland Lehoucq ${ }^{2}$, Jean-Pierre \\ Luminet $^{1}$, Jean-Philippe Uzan ${ }^{3}$ and Jeffrey Weeks ${ }^{4}$ \\ (1) Département d'Astrophysique Relativiste et de Cosmologie, \\ Observatoire de Paris - C.N.R.S. UMR 8629, F-92195 Meudon Cedex (France). \\ (2) CE-Saclay, DSM/DAPNIA/Service d'Astrophysique, \\ F-91191 Gif sur Yvette Cedex (France) \\ (3) Laboratoire de Physique Théorique - C.N.R.S. UMR 8627, Bât. 210, \\ Université Paris XI, F-91405 Orsay Cedex (France). \\ (4) 15 Farmer St., Canton NY 13617-1120, USA.
}

\begin{abstract}
This article gives the construction and complete classification of all three-dimensional spherical manifolds, and orders them by decreasing volume, in the context of multiconnected universe models with positive spatial curvature. It discusses which spherical topologies are likely to be detectable by crystallographic methods using three-dimensional catalogs of cosmic objects. The expected form of the pair separation histogram is predicted (including the location and height of the spikes) and is compared to computer simulations, showing that this method is stable with respect to observational uncertainties and is well suited for detecting spherical topologies.
\end{abstract}

PACS numbers: 98.80.-q, 04.20.-q, 02.40.Pc

\section{Introduction}

The search for the topology of our universe has focused mainly on candidate spacetimes with locally Euclidean or hyperbolic spatial sections. This was motivated on the one hand by the mathematical simplicity of three-dimensional Euclidean manifolds, and on the other hand by observational data which had, until recently, favored a low density universe. More recently, however, a combination of astrophysical and cosmological observations (among which the luminosity distance-redshift relation up to $z \sim 1$ from type Ia supernovae [1], the cosmic microwave background (CMB) temperature anisotropies [2], gravitational lensing [3], velocity fields [4], and comoving standard rulers [5]) seems to indicate that the expansion of the universe is accelerating, with about $70 \%$ of the total energy density $\Omega_{0}$ being in the form of a dark component $\Omega_{\Lambda_{0}}$ with negative pressure, usually identified with a cosmological constant term or a quintessence field [6]. As a consequence, the spatial sections of the universe would be "almost" locally flat, i.e. their curvature radius would be larger than the horizon radius $\left(\sim 10 h^{-1} \mathrm{Gpc}\right.$, where $h$ is the Lemaitre-Hubble parameter in units of 100 $\mathrm{km} / \mathrm{s} / \mathrm{Mpc}$ ). Recent CMB measurements [2] report a first Doppler peak shifted by a few percent towards larger angular scales with respect to the peak predicted by the standard cold dark matter (CDM) inflationary model, thus favouring a marginally spherical model [7]. Indeed, under specific assumptions such as a $\Lambda-\mathrm{CDM}$ model and 
a nearly scale invariant primordial power spectrum, the value of the total energydensity parameter is given by $\Omega_{0} \equiv \Omega_{m_{0}}+\Omega_{\Lambda_{0}}=1.11_{-0.12}^{+0.13}$ to $95 \%$ confidence [8]. Note that while flat models still lie well inside the $95 \%$ confidence level, the relation between the angular diameter distance and the acoustic peak positions in the angular power spectrum makes the peak positions in models with a low matter content very dependent on small variations of the cosmological constant.

As a consequence of these observable facts, spherical spaceforms are of increasing interest for relativistic cosmology, in the framework of Friedmann-Lemaitre solutions with positive spatial curvature. Due to the current constraints on the spatial curvature of our universe and to the rigidity theorem [9], hyperbolic topologies may be too large to be detectable by crystallographic methods. For example, if $\Omega_{m_{0}}=0.3$ and $\Omega_{\Lambda_{0}}=0.6$, then even in the smallest known hyperbolic topologies the distance from a source to its nearest topological image is more than a half of the horizon radius, meaning that at least one member of each pair of topological images would lie at a redshift too high to be easily detectable. When using statistical methods for detecting a hyperbolic topology, the topological signature falls to noise level as soon as observational uncertainties are taken into account.

On the other hand, spherical topologies may be easily detectable, because for a given value of the curvature radius, spherical spaces can be as small as desired. There is no lower bound on their volumes, because in spherical geometry increasing a manifold's complexity decreases its volume, in contrast to hyperbolic geometry where increasing the complexity increases the volume. Thus many different spherical spaces would fit easily within the horizon radius, no matter how small $\Omega_{0}-1$ is.

In order to clarify some misleading terminology that is currently used in the cosmological literature, we emphasize the distinction between spherical and closed universe models. A spherical universe has spatial sections with positive curvature. The volume of each spatial section is necessarily finite, but the spacetime can be open (i.e. infinite in time) if the cosmological constant is high enough. On the other hand, a closed universe is a model in which the scale factor reaches a finite maximum value before recollapsing. It can be obtained only if the space sections are spherical and the cosmological constant is sufficiently low.

As emphasized by many authors (see e.g. [10, 11, 12, 13] for reviews), the key idea to detect the topology in a three-dimensional data set is the topological lens effect, i.e. the fact that if the spatial sections of the universe have at least one characteristic size smaller than the spatial scale of the catalog, then different images of the same object shoud appear in the survey. This idea was first implemented in the crystallographic method [14], which uses a pair separation histogram (PSH) depicting the number of pairs of the catalog's objects having the same three-dimensional spatial separations in the universal covering space. Even if numerical simulations of this method showed the appearance of spikes related to characteristic distances of the fundamental polyhedron, we proved that sharp spikes emerge only when the holonomy group has at least one Clifford translation, i.e. a holonomy that translates all points the same distance [15] (see also [16]). Since then, various generalisations of the PSH method have been proposed $[17,18,19]$ (see $[13,20]$ for a discussions of these methods) but none of them is fully satisfactory. The first step towards such a generalisation was to exploit the property that, even if there is no Clifford translation, equal distances in the universal covering space appear more often than just by chance. We thus reformulated the cosmic crystallographic method as a collecting-correlated-pairs method (CCP) [21], where the topological signal was enhanced by collecting all distance correlations in a 
single index. It was proven that this signal was relevant to detect the topology.

The goal of the present article is twofold. First we give a mathematical description of all spherical spaces, which have been overlooked in the literature on cosmology. This provides the required mathematical background for applying statistical methods to detect the topology: crystallographic methods using three-dimensional data sets such as galaxy, cluster and quasar catalogs, and methods using two-dimensional data sets such as temperature fluctuations in the Cosmic Microwave Background (CMB), and we investigate their observational signature in catalogs of discrete sources in order to complete our previous works $[14,15,20,21]$ on Euclidean and hyperbolic manifolds. We first review in $\S 2$ the basics of cosmology and topology in spherical universes, including the basic relations to deal with a Friedmann-Lemaitre universe of constant curvature and the basics of cosmic topology. We then describe and classify threedimensional spherical manifolds in $\S 3$ and $\S 4$ and explain how to use them, with full details gathered in Appendix A and Appendix B. One prediction of our former works $[15,21]$ was that the PSH method must exhibit spikes if there exists at least one Clifford translation. In $\S 5$ we discuss the spaceforms that are likely to be detectable. We show that the location and height of the spikes can be predicted analytically and then we check our predictions numerically. We also study the effect of observational errors on the stability of the PSH spectra, and briefly discuss the status of the CCP method for spherical topologies.

\section{Cosmology in a Friedmann-Lemaître spacetime with spherical spatial sections}

\subsection{Friedmann-Lemaître spacetimes of constant positive curvature}

In this section we describe the cosmology and the basics of the topology of universes with spherical spatial sections. The local geometry of such a universe is given by a Friedmann-Lemaître metric

$$
\mathrm{d} s^{2}=-\mathrm{d} t^{2}+a^{2}(t)\left(\mathrm{d} \chi^{2}+\sin ^{2} \chi \mathrm{d} \omega^{2}\right) .
$$

where $a$ is the scale factor, $t$ the cosmic time and $\mathrm{d} \omega^{2} \equiv \mathrm{d} \theta^{2}+\sin ^{2} \theta \mathrm{d} \varphi^{2}$ the infinitesimal solid angle. $\chi$ is the (dimensionless) comoving radial distance in units of the curvature radius $R_{C}$ of the 3 -sphere $S^{3}$.

The 3 -sphere $S^{3}$ can be embedded in four-dimensional Euclidean space by introducing the set of coordinates $\left(x_{\mu}\right)_{\mu=0 . .3}$ related to the intrinsic coordinates $(\chi, \theta, \varphi)$ through (see e.g. [22])

$$
\begin{aligned}
& x_{0}=\cos \chi \\
& x_{1}=\sin \chi \sin \theta \sin \varphi \\
& x_{2}=\sin \chi \sin \theta \cos \varphi \\
& x_{3}=\sin \chi \cos \theta,
\end{aligned}
$$

with $0 \leq \chi \leq \pi, 0 \leq \theta \leq \pi$ and $0 \leq \varphi \leq 2 \pi$. The 3 -sphere is then the submanifold of equation

$$
x^{\mu} x_{\mu} \equiv x_{0}^{2}+x_{1}^{2}+x_{2}^{2}+x_{3}^{2}=+1,
$$

where $x_{\mu}=\delta_{\mu \nu} x^{\nu}$. The comoving spatial distance $d$ between any two points $x$ and $y$ on $S^{3}$ can be computed using the inner product $x^{\mu} y_{\mu}$. The value of this inner product is the same in all orthonormal coordinate systems, so without loss of generality we 
may assume $x=(1,0,0,0)$ and $y=(\cos d, \sin d, 0,0)$, giving $x^{\mu} y_{\mu}=\cos d$. Hence, the comoving spatial distance between two points of comoving coordinates $x$ and $y$ is given by

$$
d[x, y]=\arccos \left[x^{\mu} y_{\mu}\right]
$$

The volume enclosed by a sphere of radius $\chi$ is, in units of the curvature radius,

$$
\operatorname{Vol}(\chi)=\pi(2 \chi-\sin 2 \chi) \text {. }
$$

A convenient way to visualize the 3 -sphere is to consider $S^{3}$ as composed of two solid balls in Euclidean space $R^{3}$, glued together along their boundaries (figure 1): each point of the boundary of one ball is the same as the corresponding point in the other ball. To represent a point of coordinates $x_{\mu}$ in a three-dimensional space we consider only the coordinates $\left(x_{i}\right)_{i=1 . .3}$, which are located in the interior of a ball, and discard the nearly redundant coordinate $x_{0}$. However, the two points of coordinates $(\chi, \theta, \phi)$ and $(\pi-\chi, \theta, \phi)$, corresponding respectively to the points $\left(x_{0}, x_{1}, x_{2}, x_{3}\right)$ and $\left(-x_{0}, x_{1}, x_{2}, x_{3}\right)$ in the four-dimensional Euclidean space, have the same coordinates $\left(x_{1}, x_{2}, x_{3}\right)$ and we thus have to use two balls, one corresponding to $0 \leq \chi \leq \pi / 2$ (i.e. $x_{0} \geq 0$ ) and the other one to $\pi / 2 \leq \chi \leq \pi$ (i.e. $x_{0} \leq 0$ ). Each ball represents half the space.

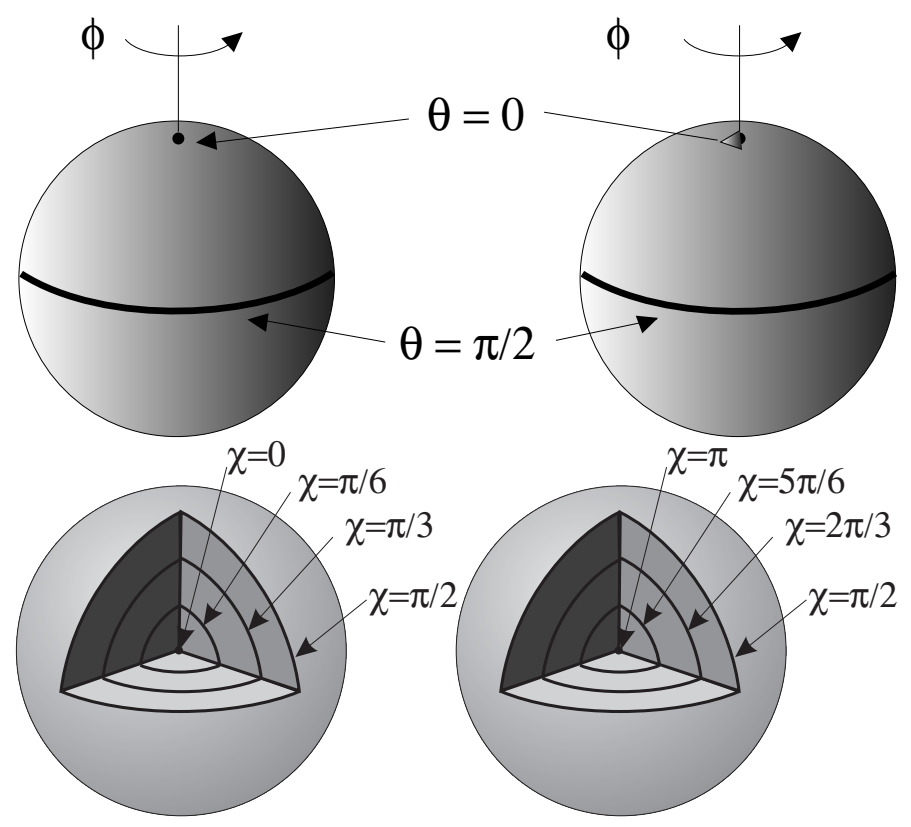

Figure 1. Representation of $S^{3}$ by two balls in $R^{3}$ glued together. Top: The $\theta$ and $\phi$ coordinates are the standard ones. Bottom: The $\chi$ coordinate runs from 0 at the center of one ball (the "north pole" of $S^{3}$ ) through $\pi / 2$ at the ball's surface (the spherical "equator" of $S^{3}$ ) to $\pi$ at the center of the other ball (the "south pole" of $\left.S^{3}\right)$.

All three-dimensional observations provide at least the position of an object on the celestial sphere and its redshift $z \equiv a / a_{0}-1$ (the value $a_{0}$ of the scale factor today may be chosen arbitrarily; a natural choice is to set $a_{0}$ equal to the physical curvature radius today). To reconstruct an object's three-dimensional position $(\chi, \theta, \varphi)$ we need 
to compute the relation between the radial coordinate $\chi$ and the redshift $z$, which requires the law of evolution of the scale factor obtained from the Friedmann equations

$$
\begin{aligned}
& H^{2}=\frac{\kappa}{3} \rho-\frac{k}{a^{2} R_{C}^{2}}+\frac{\Lambda}{3} \\
& \frac{\ddot{a}}{a}=-\frac{\kappa}{6}(\rho+3 P)+\frac{\Lambda}{6}
\end{aligned}
$$

where $\rho$ and $P$ are the energy density and pressure of the cosmic fluid, $\Lambda$ the cosmological constant, $\kappa \equiv 8 \pi G$ with $G$ the Newton constant, $k=+1$ is the curvature index and $H \equiv \dot{a} / a$ is the Hubble parameter. As a first consequence, we deduce from these equations that the physical curvature radius today is given by

$$
R_{C_{0}}^{\text {phys }} \equiv a_{0} R_{C_{0}}=\frac{c}{H_{0}} \frac{1}{\sqrt{\left|\Omega_{\Lambda_{0}}+\Omega_{m_{0}}-1\right|}}
$$

where the density parameters are defined by

$$
\Omega_{\Lambda} \equiv \frac{\Lambda}{3 H^{2}} \quad \Omega_{m} \equiv \frac{\kappa \rho}{3 H^{2}} .
$$

As emphasized above, we can choose $a_{0}$ to be the physical curvature radius today, i.e. $a_{0}=R_{C_{0}}^{\text {phys }}$, which amounts to choosing the units on the comoving sphere such that $R_{C_{0}}=1$, hence determining the value of the constant $a_{0}$. As long as we are dealing with catalogs of galaxies or clusters, we can assume that the universe is filled with a pressureless fluid. Integrating the radial null geodesic equation $\mathrm{d} \chi=\mathrm{d} t / a$ leads to

$$
\chi(z)=\int_{0}^{z} \frac{\sqrt{\Omega_{m_{0}}+\Omega_{\Lambda_{0}}-1} \mathrm{~d} x}{\sqrt{\Omega_{\Lambda_{0}}+\left(1-\Omega_{m_{0}}-\Omega_{\Lambda_{0}}\right)(1+x)^{2}+\Omega_{m_{0}}(1+x)^{3}}} .
$$

\subsection{Basics of cosmic topology}

Equations (1)-(10) give the main properties that describe the local geometry, i.e. the geometry of the universal covering space $\Sigma$, independently of the topology. Indeed it is usually assumed that space is simply connected so that the spatial sections are the 3 -sphere $S^{3}$. To describe the topology of these spatial sections we have to introduce some basic topological elements (see [10] for a review). From a topological point of view, it is convenient to describe a three-dimensional multi-connected manifold $M$ by its fundamental polyhedron, which is convex with an even number of faces. The faces are identified by face-pairing isometries. The face-pairing isometries generate the holonomy group $\Gamma$, which acts without fixed points on the three-dimensional covering space $\Sigma$ (see [22, 23, 24] for mathematical definitions and [10,13] for an introduction to topology in the cosmological context). The holonomy group $\Gamma$ is isomorphic to the first fundamental group $\pi_{1}(M)$.

To illustrate briefly these definitions, let us consider the particularly simple case of a two-dimensional flat torus $T^{2}$. It can be constructed from a square, opposite edges of which are glued together. The translations taking one edge to the other are the face pairing isometries. The holonomy group of this space, generated by the facepairing translations, is isomorphic to the group of loops on the torus $\pi_{1}\left(T^{2}\right)=Z \times Z$ (see figure 2).

In our case $\Sigma=S^{3}$ and its isometry group is the rotation group of the fourdimensional space in which it sits, i.e. $G=S O(4)$. In units of the curvature radius, the volume of the spatial sections is given by

$$
\operatorname{Vol}\left(S^{3} / \Gamma\right)=\frac{\operatorname{Vol}\left(S^{3}\right)}{|\Gamma|}=\frac{2 \pi^{2}}{|\Gamma|},
$$


where $|\Gamma|$ is the order of the group $\Gamma$, i.e. the number of elements contained in $\Gamma$. Since the elements $g$ of $\Gamma$ are isometries, they satisfy

$$
\forall x, y \in \Sigma \quad \forall g \in \Gamma, \quad \operatorname{dist}[x, y]=\operatorname{dist}[g(x), g(y)]
$$

An element $g$ of $\Gamma$ is a Clifford translation if it translates all points the same distance, i.e. if

$$
\forall x, y \in \Sigma \quad \operatorname{dist}[x, g(x)]=\operatorname{dist}[y, g(y)]
$$

The significance of these particular holonomies, which are central to the detection of the topology, will be explained in section 3.1. To perform computations we express the isometries of the holonomy group $\Gamma \subset S O(4)$ as $4 \times 4$ matrices. To enumerate all spherical manifolds we will need a classification of all finite, fixed-point free subgroups of $S O(4)$. This will be the purpose of sections 3 and 4 .
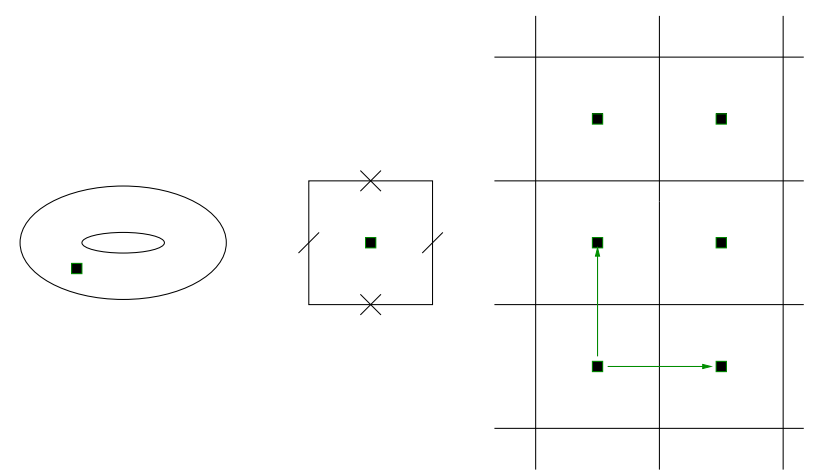

Figure 2. Illustration of the general topological definitions in the case of a twodimensional torus (left). Its fundamental polyhedron is a square, opposite faces of which are identified (middle). In its universal covering space (right) the two translations taking the square to its nearest images generate the holonomy group.

\subsection{Spherical spaces and cosmology}

As early as 1917, de Sitter [25] distinguished the sphere $S^{3}$ from the projective space $P^{3}$ (which he called elliptical space) in a cosmological context. Both spaceforms are finite, with (comoving) volumes $2 \pi^{2}$ and $\pi^{2}$, respectively. The projective space $P^{3}$ is constructed from the sphere $S^{3}$ by identifying all pairs of antipodal points. The main difference between them is that in a sphere all straight lines starting from a given point reconverge at the antipodal point, whereas in projective space two straight lines can have at most one point in common. Thus the sphere does not satisfy Euclid's first axiom, while projective space does. In $S^{3}$ the maximal distance between any two points is $\pi$, and from any given point there is only one point, the antipodal one, at that maximal distance. In $P^{3}$ the maximal distance is $\pi / 2$ and the set of points lying at maximal distance from a given point forms a two-dimensional projective plane $P^{2}$. Because each pair of antipodal point points in the 3 -sphere projects to a single point in projective space, if we adopt the 3 -sphere as our space model we may be representing the physical world in duplicate. For that reason, de Sitter claimed that $P^{3}$ was really the simplest case, and that it was preferable to adopt this in a cosmological context. 
Einstein did not share this opinion, and argued that the simple connectivity of $S^{3}$ was physically preferable [26]. Eddington [27], Friedmann [28] and Lemaître [29] also referred to projective space as a more physical alternative to $S^{3}$.

Narlikar and Seshadri [30] examined the conditions under which ghost images of celestial objects may be visible in a Friedmann-Lemaitre model with the topology of projective space. De Sitter had correctly remarked that the most remote points probably lie beyond the horizon, so that the antipodal point, if any, would remain unobservable. Indeed, given the present data, it will be impossible to distinguish $S^{3}$ from $P^{3}$ observationally if $\Omega_{0}-1 \ll 1$, because our horizon radius is too small.

None of these authors mentioned other multi-connected spaces in a cosmological context. This was first done by Ellis [31] and Gott [32]. More recently, lens spaces have been investigated in the framework of quantum gravity models [33] and in terms of their detectability [34]. Nevertheless, the literature on multi-connected spherical cosmologies is underdeveloped; here we aim to fill the gap.

\section{The Mathematics of Spherical Spaces I: Classification of $S^{3}$ subgroups}

This section describes the classification of all spherical 3-manifolds and develops an intuitive understanding of their topology and geometry. Of particular observational relevance, we will see which spherical 3-manifolds have Clifford translations in their holonomy groups and which do not. Threlfall and Seifert [35] gave the first complete classification of these spherical 3-manifolds. Our approach borrows heavily from theirs, while also making use of quaternions as in [36].

As emphasized in the previous section, technically what we will need in order to perform any computation is the form of the holonomy transformations $g$ as $4 \times 4$ matrices in $S O(4)$, and an enumeration of all finite subgroups $\Gamma \subset S O(4)$. We will enumerate the finite subgroups of $S O(4)$ (see sections 3.1 and 4 ) in terms of the simpler enumeration of finite subgroups of $S O(3)$ (see section 3.2). The connection between $S O(4)$ and $S O(3)$ will use quaternions (see Appendix A and Appendix B).

\subsection{Generalities}

Our starting point is the fact that for each isometry $g \in O(n+1)$ there is a basis of $R^{n+1}$ relative to which the matrix of $g$ has the form

$$
\left(\begin{array}{ccccccccc}
+1 & 0 & \cdots & & & & & & \\
0 & \ddots & & & & & & & \\
& & +1 & & & & & & \\
\vdots & & & -1 & & & & & \\
& & & & \ddots & & & & \\
& & & & & -1 & & & \\
& & & & & & \cos \alpha & -\sin \alpha & \\
& & & & & & \sin \alpha & \cos \alpha & \\
& & & & & & & & \ddots
\end{array}\right)
$$

If $g$ is a holonomy transformation of an $n$-manifold, then $g$ has no fixed points and its matrix has no +1 terms on the diagonal. Furthermore, each pair of -1 terms may be 
rewritten as a sine- $\operatorname{cosine}$ block with $\alpha=\pi$. Thus when $n=3$ the matrix takes the form

$$
M(\theta, \phi)=\left(\begin{array}{cccc}
\cos \theta & -\sin \theta & 0 & 0 \\
\sin \theta & \cos \theta & 0 & 0 \\
0 & 0 & \cos \phi & -\sin \phi \\
0 & 0 & \sin \phi & \cos \phi
\end{array}\right)
$$

An immediate consequence of this decomposition is that every spherical 3-manifold is orientable. Indeed all odd-dimensional spherical manifolds must be orientable for this same reason. In even dimensions the only spherical manifolds are the $n$-sphere $S^{n}$ (which is orientable) and the $n$-dimensional projective space $P^{n}$ (which is non orientable).

If we replace the static matrix $M(\theta, \phi)$ with the time-dependent matrix $M(\theta t, \phi t)$, we generate a flow on $S^{3}$, i.e. to each point $x \in S^{3}$ we associate the flow line $x(t)=M(\theta t, \phi t) x$. This flow is most beautiful in the special case $\theta= \pm \phi$. In this special case all flow lines are geodesics (great circles), and the flow is homogeneous in the sense that there is an isometry of $S^{3}$ taking any flow line to any other flow line. The matrix $M(\theta, \pm \theta)$ defines a Clifford translation because it translates all points the same distance (see equation 13). We further distinguish two families of Clifford translations. When $\theta=\phi$ the flow lines spiral clockwise around one another, and the Clifford translation is considered right-handed whereas when $\theta=-\phi$ the flow lines spiral anticlockwise around one another, and the Clifford translation is considered left-handed.

Every isometry $M(\theta, \phi) \in S O(4)$ is the product of a right-handed Clifford translation $M(\alpha, \alpha)$ and a left-handed Clifford translation $M(\beta,-\beta)$ as

$$
M(\theta, \phi)=M(\alpha, \alpha) M(\beta,-\beta)=M(\beta,-\beta) M(\alpha, \alpha)
$$

where $\alpha \equiv(\theta+\phi) / 2$ and $\beta \equiv(\theta-\phi) / 2$ and the order of the factors makes no difference. This factorization is unique up to simultaneously multiplying both factors by -1 . Moreover every right-handed Clifford translation commutes with every lefthanded one, because there is always a coordinate system that simultaneously brings both into their canonical form (15).

Just as the unit circle $S^{1}$ enjoys a group structure as the set $\mathcal{S}^{1}$ of complex numbers of unit length, the 3 -sphere $S^{3}$ enjoys a group structure as the set $\mathcal{S}^{3}$ of quaternions of unit length (see Appendix A for details). Each right-handed Clifford translation corresponds to left multiplication by a unit length quaternion $(\mathbf{q} \rightarrow \mathbf{x q})$, so the group of all right-handed Clifford translations is isomorphic to the group $\mathcal{S}^{3}$ of unit length quaternions, and similarly for the left-handed Clifford translations, which correspond to right multiplication $(\mathbf{q} \rightarrow \mathbf{q x})$. It follows that $S O(4)$ is isomorphic to $\mathcal{S}^{3} \times \mathcal{S}^{3} /\{ \pm(\mathbf{1}, \mathbf{1})\}$, where $\mathbf{1}$ is the identity quaternion, so the classification of all subgroups of $S O(4)$ can be deduced from the classification of all subgroups of $\mathcal{S}^{3}$.

Classifying all finite subgroups of $\mathcal{S}^{3}$ seems difficult at first, but luckily it reduces to a simpler problem. The key is to consider the action of the quaternions by conjugation. That is, for each unit length quaternion $\mathrm{x} \in \mathcal{S}^{3}$, consider the isometry $p_{\mathbf{x}}$ that sends each quaternion $\mathbf{q}$ to $\mathbf{x q} \mathbf{x}^{-1}$

$$
p_{\mathbf{x}}:\left\{\begin{array}{l}
\mathcal{S}^{3} \rightarrow \mathcal{S}^{3} \\
\mathbf{q} \longmapsto p_{\mathbf{x}}(\mathbf{q})=\mathbf{x q x}^{-1}
\end{array}\right.
$$

The isometry $p_{\mathbf{x}}$ fixes the identity quaternion $\mathbf{1}$, so in effect its action is confined to the equatorial 2-sphere spanned by the remaining basis quaternions $(\mathbf{i}, \mathbf{j}, \mathbf{k})$ [see Appendix 
A for details and definitions concerning quaternions]. Thus, by restricting our attention to the equatorial 2-sphere, we get an isometry

$$
p_{\mathbf{x}}: S^{2} \rightarrow S^{2}
$$

In other words, each $\mathbf{x} \in \mathcal{S}^{3}$ defines an element $p_{\mathbf{x}} \in S O(3)$, and the mapping

$$
p:\left\{\begin{array}{l}
\mathcal{S}^{3} \rightarrow S O(3) \\
\mathbf{x} \longmapsto p(\mathbf{x})=p_{\mathbf{x}}
\end{array}\right.
$$

is a homomorphism from $\mathcal{S}^{3}$ to $S O(3)$. To classify all subgroups of $\mathcal{S}^{3}$ we must first know the finite subgroups of $S O(3)$.

\subsection{Finite Subgroups of $S O(3)$}

The finite subgroups of $S O(3)$ are just the finite rotation groups of a 2-sphere, which are known to be precisely the following:

- The cyclic groups $Z_{n}$ of order $n$, generated by a rotation through an angle $2 \pi / n$ about some axis.

- The dihedral groups $D_{m}$ of order $2 m$, generated by a rotation through an angle $2 \pi / m$ about some axis as well as a half turn about some perpendicular axis.

- The tetrahedral group $T$ of order 12 consisting of all orientation-preserving symmetries of a regular tetrahedron.

- The octahedral group $O$ of order 24 consisting of all orientation-preserving symmetries of a regular octahedron.

- The icosahedral group I of order 60 consisting of all orientation-preserving symmetries of a regular icosahedron.

If the homomorphism $p: \mathcal{S}^{3} \rightarrow S O(3)$ were an isomorphism, the above list would give the finite subgroups of $\mathcal{S}^{3}$ directly. We are not quite that lucky, but almost: the homomorphism $p$ is two-to-one. It is easy to see that $p_{\mathbf{x}}=p_{-\mathbf{x}}$ because $\mathbf{x q x ^ { - 1 }}=(-\mathbf{x}) \mathbf{q}(-\mathbf{x})^{-1}$ for all $\mathbf{q}$. There are no other redundancies, so the kernel of $p$ is

$$
\operatorname{Ker}(p)=\{ \pm \mathbf{1}\}
$$

Let $\Gamma$ be a finite subgroup of $\mathcal{S}^{3}$ and consider separately the cases that $\Gamma$ does or does not contain $\mathbf{- 1}$.

- Case 1: If $\Gamma$ does not contain -1, then $p$ maps $\Gamma$ one-to-one onto its image in $S O(3)$, and $\Gamma$ is isomorphic to one of the groups in the above list. Moreover, because we have excluded $\mathbf{- 1}$, and $\mathcal{S}^{3}$ contains no other elements of order 2 , we know that $\Gamma$ contains no elements of order 2 . The only groups on the above list without elements of order 2 are the cyclic groups $Z_{n}$ of odd order. Thus only cyclic groups of odd order may map isomorphically from $\mathcal{S}^{3}$ into $S O(3)$, and it is easy to check that they all do, for example by choosing the generator of $Z_{n}$ to be the quaternion $\cos (2 \pi / n) \mathbf{1}+\sin (2 \pi / n)$ i.

- Case 2: If $\Gamma$ contains $\mathbf{- 1}$, then $p$ maps $\Gamma$ two-to-one onto its image in $S O(3)$, and $\Gamma$ is a two-fold cover of one of the groups in the above list. Conversely, every group on the list lifts to a group $\Gamma \subset \mathcal{S}^{3}$. The construction of the two-fold cover is trivially easy: just take the preimage of the group under the action of $p$. The result is called the binary cyclic, binary dihedral, binary tetrahedral, binary octahedral, 
or binary icosahedral group. A "binary cyclic group" is just a cyclic group of twice the order, so all even order cyclic groups occur in this fashion. The remaining binary groups are not merely the product of the original polyhedral group with a $Z_{2}$ factor, nor are they isomorphic to the so-called extended groups which include the orientation-reversing as well as the orientation-preserving symmetries of the given polyhedron, but are something completely new $\ddagger$. Note that the plain dihedral, tetrahedral, octahedral, and icosahedral groups do not occur as subgroups as of $\mathcal{S}^{3}$ - only their binary covers do.

\subsection{Finite Subgroups of $\mathcal{S}^{3}$}

Combining the results of the two previous cases, we get the complete classification of finite subgroups of the group $\mathcal{S}^{3}$ of unit length quaternions as follows:

- The cyclic groups $Z_{n}$ of order $n$.

- The binary dihedral groups $D_{m}^{*}$ of order $4 m, m \geq 2$.

- The binary tetrahedral group $T^{*}$ of order 24 .

- The binary octahedral group $O^{*}$ of order 48.

- The binary icosahedral group $I^{*}$ of order 120 .

\section{The Mathematics of Spherical Spaces II: Classification of spherical spaceforms}

There are three categories of spherical 3-manifolds. The single action manifolds are those for which a subgroup $R$ of $\mathcal{S}^{3}$ acts as pure right-handed Clifford translations. The double action manifolds are those for which subgroups $R$ and $L$ of $\mathcal{S}^{3}$ act simultaneously as right- and left-handed Clifford translations, and every element of $R$ occurs with every element of $L$. The linked action manifolds are similar to the double action manifolds, except that each element of $R$ occurs with only some of the elements of $L$.

After introducing some definitions, we give the classifications of single action manifolds (§ 4.1), double action manifolds (§ 4.2) and linked action manifolds (§ 4.3) and present in $\S 4.4$ a summary of these classifications.

We define a lens space $L(p, q)$ by identifying the lower surface of a lens-shaped solid to the upper surface with a $2 \pi q / p$ rotation (see figure 3 ), for relatively prime integers $p$ and $q$ with $0<q<p$. Furthermore, we may restrict our attention to $0<q \leq p / 2$ because for values of $q$ in the range $p / 2<q<p$ the twist $2 \pi q / p$ is the same as $-2 \pi(p-q) / p$, thus $L(p, q)$ is the mirror image of $L(p, p-q)$. When the lens is drawn in Euclidean space its faces are convex, but when it is realized in the 3 -sphere its faces lie on great 2 -spheres, filling a hemisphere of each. Exactly $p$ copies of the lens tile the universal cover $S^{3}$, just as the 2-dimensional surface of an orange may be tiled with $p$ sections of orange peel, each of which is a bigon with straight sides meeting at the poles. Two lens spaces $L(p, q)$ and $L\left(p^{\prime}, q^{\prime}\right)$ are homeomorphic if and only if $p=p^{\prime}$ and either $q= \pm q^{\prime}(\bmod p)$ or $q q^{\prime}= \pm 1(\bmod p)$.

A cyclic group $Z_{n}$ may have several different realizations as holonomy groups $\Gamma \subset S O(4)$. For example, the lens spaces $L(5,1)$ and $L(5,2)$ are nonhomeomorphic manifolds, even though their holonomy groups are both isomorphic to $Z_{5}$. For

$\ddagger$ The binary dihedral group $D_{1}^{*}$ is isomorphic to the plain cyclic group $Z_{4}$. 
noncyclic groups, the realization as a holonomy group $\Gamma \subset S O(4)$ is unique up to an orthonormal change of basis, and thus the resulting manifold is unique.

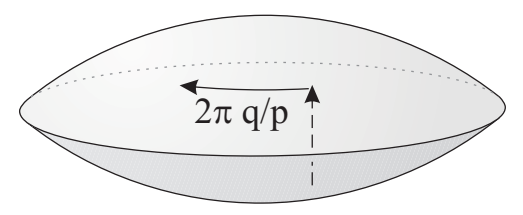

Figure 3. Construction of a lens space $L(p, q)$.

\subsection{Single Action Spherical 3-Manifolds}

The finite subgroups of $\mathcal{S}^{3}$ give the single action manifolds directly, which are thus the simplest class of spherical 3-manifolds. They are all given as follows:

- Each cyclic group $Z_{n}$ gives a lens space $L(n, 1)$, whose fundamental domain is a lens shaped solid, $n$ of which tile the 3-sphere.

- Each binary dihedral group $D_{m}^{*}$ gives a prism manifold, whose fundamental domain is a $2 m$-sided prism, $4 m$ of which tile the 3 -sphere.

- The binary tetrahedral group $T^{*}$ gives the octahedral space, whose fundamental domain is a regular octahedron, 24 of which tile the 3 -sphere in the pattern of a regular 24-cell.

- The binary octahedral group $O^{*}$ gives the truncated cube space, whose fundamental domain is a truncated cube, 48 of which tile the 3 -sphere.

- The binary icosahedral group $I^{*}$ gives the Poincaré dodecahedral space, whose fundamental domain is a regular dodecahedron, 120 of which tile the 3-sphere in the pattern of a regular 120-cell. Poincaré discovered this manifold in a purely topological context, as the first example of a multiply connected homology sphere [37]. A quarter century later Weber and Seifert glued opposite faces of a dodecahedron and showed that the resulting manifold was homeomorphic to Poincaré's homology sphere [38].

In figure 4, we depict the fundamental domains for the binary tetrahedral group $T^{*}$, binary octahedral group $O^{*}$ and binary icosahedral group $I^{*}$. The fundamental polyhedron for the lens space $L(n, 1)$ can be constructed by following the example presented in figure 3 . Finally, the fundamental domain of the prism manifold generated by the binary dihedral group $D_{5}^{*}$ is shown in figure 5 .

To finish, let us emphasize that all single action manifolds are globally homogeneous, in the sense that there is an isometry $h$ of the manifold taking any point $x$ to any other point $y$. If the manifold's holonomy group is realized as left multiplication by a group $\Gamma=\left\{g_{i}\right\}$ of quaternions, then the isometry $h$ is realized as right multiplication by $x^{-1} y$. To check that $h$ is well-defined on the quotient manifold $S^{3} / \Gamma$, note that $h$ takes any point $g_{i} x$ equivalent to $x$ to a point $g_{i} x\left(x^{-1} y\right)=g_{i} y$ equivalent to $y$, thus respecting equivalence classes of points.

\subsection{Double action spherical 3-manifolds}

The double action spherical 3-manifolds are obtained by letting one finite subgroup $R \subset \mathcal{S}^{3}$ act as right-handed Clifford translations (equivalent to left multiplication 

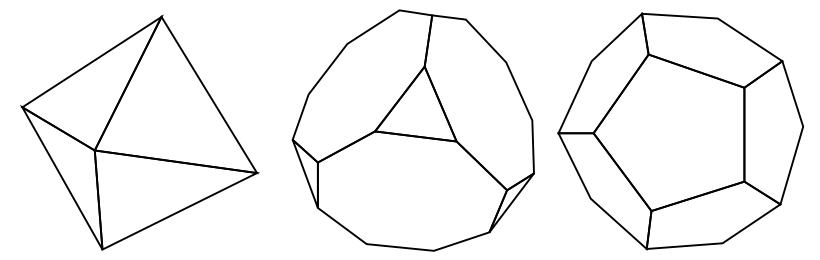

Figure 4. Fundamental domains for three single action 3-manifolds. From left to right, the regular octahedron, the truncated cube and the regular dodecahedron which respectively correspond to the spaces generated by the binary tetrahedral group $T^{*}$, the binary octahedral group $O^{*}$ and the binary icosahedral group $I^{*}$.

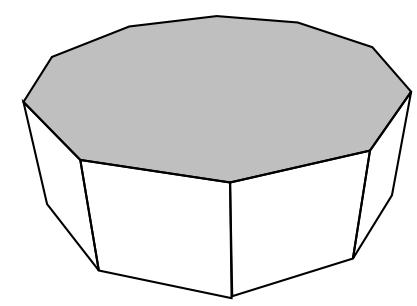

Figure 5. An example of fundamental domain for a prism manifold. This 10sided prism is the fundamental polyhedron of the space generated by the binary dihedral group $D_{5}^{*}$, of order 20 .

of quaternions) while a different finite subgroup $L \subset \mathcal{S}^{3}$ simultaneously acts as lefthanded Clifford translations (equivalent to right multiplication of quaternions). A priori any two subgroups of $\mathcal{S}^{3}$ could be used. However, if an element $M(\theta, \theta)$ of $R$ and an element $M(\phi,-\phi)$ of $L$ share the same translation distance $|\theta|=|\phi|$, then their composition, which equals $M(2 \theta, 0)$ or $M(0,2 \theta)$, will have fixed points unless $\theta=\phi=\pi$. Allowing fixed points would take us into the realm of orbifolds, which most cosmologists consider unphysical to describe our universe, so we do not consider them here. In practice this means that the groups $R$ and $L$ cannot contain elements of the same order, with the possible exception of $\pm \mathbf{1}$. The binary dihedral, tetrahedral, octahedral, and icosahedral groups all contain elements of order 4, and so cannot be paired with one another.

Thus either $R$ or $L$ must be cyclic. Without loss of generality we may assume that $L$ is cyclic. If $R$ is also cyclic, then $L$ and $R$ cannot both contain elements of order 4 , and so we may assume that it is $L$ that has no order 4 elements. If $R$ is not cyclic, then $R$ is a binary polyhedral group, and again $L$ can have no elements of order 4. Thus either $L=Z_{n}$ or $L=Z_{2 n}$, with $n$ odd. Recalling that the group $Z_{n}$ consists of the powers $\left\{q^{i}\right\}_{0 \leq i<n}$ of a quaternion $q$ of order $n$, it's convenient to think of the group $Z_{2 n}$ as $\left\{q^{i}\right\}_{0 \leq i<n} \cup\left\{-q^{i}\right\}_{0 \leq i<n}$. If $R$ contains -1, then nothing is gained by including the $\left\{-q^{i}\right\}$ in $L$, because each possible element $(r)(-l)$ already occurs as $(-r)(l)$. In other words, $L=Z_{n}$ and $L=Z_{2 n}$ produce the same result, the only difference being that with $L=Z_{2 n}$ each element of the resulting group is generated twice, once as $(r)(l)$ and once as $(-r)(-l)$. If $R$ does not contain $-\mathbf{1}$, then it is cyclic of odd order and we swap the roles of $R$ and $L$. Either way, we may assume $L$ is cyclic of odd order. The double action spherical 3-manifolds are therefore the following:

- $R=Z_{m}$ and $L=Z_{n}$, with $m$ and $n$ relatively prime, always yields a lens space 
$L(m n, q)$. However, not all lens spaces arise in this way.

- $R=D_{m}^{*}$ and $L=Z_{n}$, with $\operatorname{gcd}(4 m, n)=1$, yields an $n$-fold quotient of a prism manifold that is simultaneously a $4 m$-fold quotient of the lens space $L(n, 1)$.

- $R=T^{*}$ and $L=Z_{n}$, with $\operatorname{gcd}(24, n)=1$, yields an $n$-fold quotient of the octahedral space that is simultaneously a 24 -fold quotient of the lens space $L(n, 1)$.

- $R=O^{*}$ and $L=Z_{n}$, with $\operatorname{gcd}(48, n)=1$, yields an $n$-fold quotient of the truncated cube space that is simultaneously a 48 -fold quotient of the lens space $L(n, 1)$.

- $R=I^{*}$ and $L=Z_{n}$, with $\operatorname{gcd}(120, n)=1$, yields an $n$-fold quotient of the Poincaré dodecahedral space that is simultaneously a 120 -fold quotient of the lens space $L(n, 1)$.

In figure 6 , we present the fundamental domain of the double action manifold generated by the binary octahedral group $R=O^{*}$ and the cyclic group $L=Z_{5}$, of orders 48 and 5 respectively.

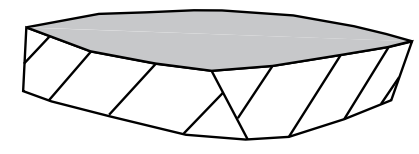

Figure 6. A fundamental domain for the double action manifold of order 240 generated by the binary octahedral group $R=O^{*}$ and the cyclic group $L=Z_{5}$.

\subsection{Linked Action Spherical 3-Manifolds}

The third and final way to construct spherical $3-$ manifolds is to choose groups $R$ and $L$ as before, but allow each element $r \in R$ to pair with a restricted class of elements $l \in L$, being careful to exclude combinations of $r$ and $l$ that would create fixed points. The following linked action manifolds arise in this way.

- $R$ and $L$ are the cyclic groups respectively generated by

$$
r=M\left(\frac{p+q+1}{2 p} 2 \pi, \frac{p+q+1}{2 p} 2 \pi\right)
$$

and

$$
l=M\left(\frac{p-q+1}{2 p} 2 \pi,-\frac{p-q+1}{2 p} 2 \pi\right)
$$

with $0<q<p$ and $\operatorname{gcd}(p, q)=1$. The generator $r$ is linked to the generator $l$, and their powers are linked accordingly. This yields the lens space $L(p, q)$, generated by $r l=M(2 \pi / p, 2 \pi q / p)$. Note that when $p+q$ is even, each element of $L(p, q)$ is produced twice, once as $r^{k} l^{k}$ and once as $r^{p+k} l^{p+k}=r^{p} r^{k} l^{p} l^{k}=\left(-r^{k}\right)\left(-l^{k}\right)$.

- $R=T^{*}$ and $L=Z_{9 n}$ with $n$ odd. The plain (not binary) tetrahedral group $T$ contains a normal subgroup $H \simeq D_{2}$ consisting of the three half turns plus the identity. Each element $r$ in the binary tetrahedral group $T^{*}$ is assigned an index 0,1 , or 2 according to the coset of $H$ in which its projection $p_{r} \in S O(3)$ lies. Each element $l$ in $Z_{9 n}$ is assigned an index 0,1 , or 2 equal to its residue modulo 3. 
An element $r$ is linked to an element $l$ if and only if their indices are equal. This yields a holonomy group with only a third as many elements as the full double action group would have, and avoids elements with fixed points.

- $R=D_{m}^{*}$ and $L=Z_{8 n}$ with $\operatorname{gcd}(m, 8 n)=1$. Each element $r$ in $D_{m}^{*}$ is assigned an index 0 or 1 according to whether its projection $p_{r} \in S O(3)$ lies in the cyclic part of the plain $D_{m}$ or not. Each element $l$ in $Z_{8 n}$ is assigned an index 0 or 1 equal to its residue modulo 2. An element $r$ is linked to an element $l$ if and only if their indices are equal. This yields a holonomy group with only half as many elements as the full double action group would have, and avoids elements with fixed points. Note that because $R$ and $L$ both contain $-\mathbf{1}$, each element in the group is produced twice, once as $r l$ and once as $(-r)(-l)$.

As an example, figure 7 shows the fundamental polyhedron of the linked action manifold generated by the binary tetrahedral group $R=T^{*}$ and the cyclic group $L=Z_{9}$, of orders 24 and 9 respectively.

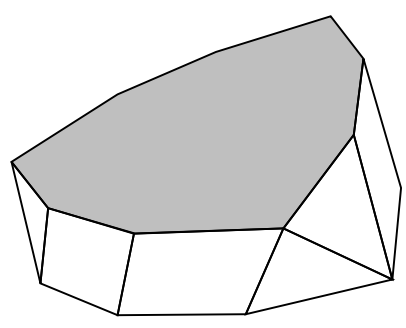

Figure 7. Fundamental domain of the linked action manifold of order 72 generated by the binary tetrahedral group $R=T^{*}$ and the cyclic group $L=Z_{9}$.

\subsection{Summary of classification}

The preceding sections constructed all three-dimensional spherical manifolds and classified them in three families. To sum up, figure 8 organizes these manifolds by decreasing volume. Since none homeomorphic lens spaces can have isomorphic holonomy group, figure 9 charts this special case of lens spaces.

On Figure 10, we show the number of distinct spherical 3-manifolds of a given order. The vast majority of these manifolds are lens spaces. This does not necessarily mean that a spherical universe is "more likely" to be a lens space. It does, however, reflect the fact that there is a free parameter governing the amount of twist in the construction of a lens space of order $n$. The remaining groups of order $n$ have no free parameters in their construction. The difference arises because each lens space is generated by a single element and is therefore subject to minimal consistency constraints; the remaining manifolds, which each requires at least two generators, are subject to stronger consistency conditions.

In the construction of a lens space of order $n$, there are roughly $n$ choices for the amount of twist (the exact number of choices varies with $n$, because the twist parameter must be relatively prime to $n$ ), so the number of distinct spherical manifolds of order exactly $n$ grows linearly with $n$ (see Figure 10, left plot). Thus the total number of spherical 3-manifolds of order $n$ or less is the sum of an arithmetic series, and therefore grows quadratically with $n$ (see Figure 10, right plot). 


\begin{tabular}{|c|c|c|c|}
\hline Order & Single action & Double action & Linked action \\
\hline 1 & $Z_{1}$ & & \\
\hline 2 & $Z_{2}$ & & \\
\hline 3 & $Z_{3}$ & & \\
\hline 4 & $Z_{4}$ & & \\
\hline 5 & $Z_{5}$ & & $Z_{5}$ \\
\hline 6 & $Z_{6}$ & & \\
\hline 7 & $Z_{7}$ & & $Z_{7}$ \\
\hline 8 & $Z_{8}$ & & $Z_{8}$ \\
\hline$\ldots$ & & & \\
\hline 12 & $Z_{12} \quad D_{3}^{*}$ & $Z_{3} \times Z_{4}$ & \\
\hline$\ldots$ & & & \\
\hline 72 & $\begin{array}{ll}Z_{72} & D_{18}^{*}\end{array}$ & $Z_{8} \times Z_{9} \quad D_{2}^{*} \times Z_{9}$ & $\begin{array}{cc}Z_{72} & T^{*} \times Z_{9} \\
D_{9}^{*} \times Z_{8} & \\
\end{array}$ \\
\hline$\ldots$ & & & \\
\hline 120 & $\begin{array}{cc}Z_{120} & D_{30}^{*} \\
I^{*} & \end{array}$ & $\begin{array}{ccc}Z_{40} \times Z_{3} & Z_{24} \times Z_{5} & Z_{8} \times Z_{15} \\
D_{10}^{*} \times Z_{3} & D_{6}^{*} \times Z_{5} & D_{2}^{*} \times Z_{15} \\
T^{*} \times Z_{5} & & \\
\end{array}$ & $\begin{array}{cl}Z_{120} & D_{3}^{*} \times Z_{40} \\
D_{5}^{*} \times Z_{24} & D_{15}^{*} \times Z_{8}\end{array}$ \\
\hline$\ldots$ & & & \\
\hline 216 & $Z_{216} \quad D_{54}^{*}$ & $Z_{8} \times Z_{27} \quad D_{2}^{*} \times Z_{27}$ & $\begin{array}{cc}Z_{216} & T^{*} \times Z_{27} \\
D_{27}^{*} \times Z_{8} & \\
\end{array}$ \\
\hline$\ldots$ & & & \\
\hline 240 & $\begin{array}{ll}Z_{240} & D_{60}^{*}\end{array}$ & $\begin{array}{ccc}Z_{80} \times Z_{3} & Z_{48} \times Z_{5} & Z_{16} \times Z_{15} \\
D_{3}^{*} \times Z_{80} & D_{12}^{*} \times Z_{5} & D_{4}^{*} \times Z_{15} \\
O^{*} \times Z_{5} & & \\
\end{array}$ & $\begin{array}{cc}D_{20}^{*} \times Z_{3} & Z_{240} \\
D_{5}^{*} \times Z_{48} & D_{15}^{*} \times Z_{16}\end{array}$ \\
\hline
\end{tabular}

Figure 8. Classification of groups generating single, double and linked action spherical 3-manifolds. The first column gives the order $|\Gamma|$ of the holonomy group, i.e. along each row the volume of space is $2 \pi^{2} /|\Gamma|$. The simply connected 3-sphere is $S^{3} / Z_{1}$, and the projective space $P^{3}$ is $S^{3} / Z_{2}$. Since some cyclic groups $Z_{n}$ have different realizations as single action, double action, and linked action manifolds they may appear more than once on a given line. For example, the lens spaces $L(5,1)$ and $L(5,2)$ are nonhomeomorphic manifolds, even though their holonomy groups are both isomorphic to $Z_{5}$. The double action groups $Z_{m} \times Z_{n} \simeq Z_{m n}$ all yield lens spaces, as do the single action groups $Z_{m n}$. For instance $L(12,1)$ is the single action manifold generated by $Z_{12}$ while $L(12,5)$ is the double action manifold generated by $Z_{3} \times Z_{4} \simeq Z_{12}$. For noncyclic groups, the realization as a holonomy group is unique, and thus the resulting manifold is unique, named after its holonomy group.

\section{Crystallographic Simulations}

As we emphasized, the PSH method applies as long as the holonomy group has at least one Clifford translation. Thus we use the PSH method for most of the spherical manifolds but we will need the CCP method in certain exceptional cases.

Section 5.1 determines the radius $\chi_{\max }$ of the observable portion of the covering 3sphere in units of the curvature radius, as a function of the cosmological parameters $\Omega_{m}$ and $\Omega_{\Lambda}$ and a redshift cutoff $z_{c}$. For plausible values of $\chi_{\max }$, Section 5.2 determines which topologies are likely to be detectable. Section 5.3 explains the expected form of the Pair Separation Histogram first in the 3-sphere, and then predicts the location and 


\begin{tabular}{|c|c|c|c|}
\hline Order & Single action & Double action & Linked action \\
\hline 2 & $L(2,1)$ & & \\
\hline 3 & $L(3,1)$ & & \\
\hline 4 & $L(4,1)$ & & \\
\hline 5 & $L(5,1)$ & & \\
\hline 6 & $L(6,1)$ & & $L(7,2)$ \\
\hline 7 & $L(7,1)$ & & $L(8,3)$ \\
\hline 8 & $L(8,1)$ & & \\
\hline$\ldots$ & & & \\
\hline 12 & $L(12,1)$ & $L(12,5)$ & \\
\hline$\ldots$ & & & +5 more \\
\hline 72 & $L(72,1)$ & $L(72,17)$ & \\
& & & $+7(120,7)$ \\
\hline$\ldots$ & & & \\
\hline 120 & $L(120,1)$ & $L(120,31)$ & more \\
& & $L(120,41)$ & \\
& & $L(120,49)$ & \\
\hline$\ldots$ & & & \\
\hline 216 & $L(216,1)$ & $L(216,55)$ & $L(216,5)$ \\
& & & +17 more \\
\hline$\ldots$ & & & \\
\hline 240 & $L(240,1)$ & $L(240,31)$ & $L(240,7)$ \\
& & $L(240,41)$ & +15 more \\
& & $L(240,49)$ & \\
\hline
\end{tabular}

Figure 9. Classification of lens spaces. In this chart each lens space occurs only once in the first valid column (e.g. if the lens space is a single action manifold we ignore the trivial expression of it as a double or linked action manifold). This also takes into account the various equivalences so, for example $L(7,2)$ appears while $L(7,3)$ does not because $L(7,3)=L(7,2)$.

height of the spikes in a multiply connected spherical universe. Computer simulations $(\S 5.4)$ confirm these predictions. In $\S 5.5$, we briefly recall the applicability of the CCP method.

\subsection{Observational prospects}

Observations indicate that $\left|\Omega_{m_{0}}+\Omega_{\Lambda_{0}}-1\right|$ is at most about 0.1 , and this value fixes the physical curvature scale $R_{C_{0}}^{\text {phys }}$ from (8). To detect topology one requires that the size of the manifold be smaller than the diameter of the observable universe in at least one direction. Equation (10) gives the distance $\chi$ in radians from the observer to a source at redshift $z$. Figure 11 uses equation (10) to plot the maximal radial distance $\chi_{\max }$ that is accessible in a catalog of sources extending to redshift $z_{c}$, as a function of the cosmological parameters $\Omega_{m_{0}}$ and $\Omega_{\Lambda_{0}}$. This maximal radial distance $\chi_{\max }$ may also be used to compute the volume of the observable universe $\operatorname{vol}\left(\chi_{\max }\right)=\pi\left(2 \chi_{\max }-\sin 2 \chi_{\max }\right) \simeq \frac{4}{3} \pi \chi_{\max }^{3}$ in curvature radius units and compare it to the total volume $2 \pi^{2}$ of the 3 -sphere.

In practice one requires that shortest distance between topological images be less 

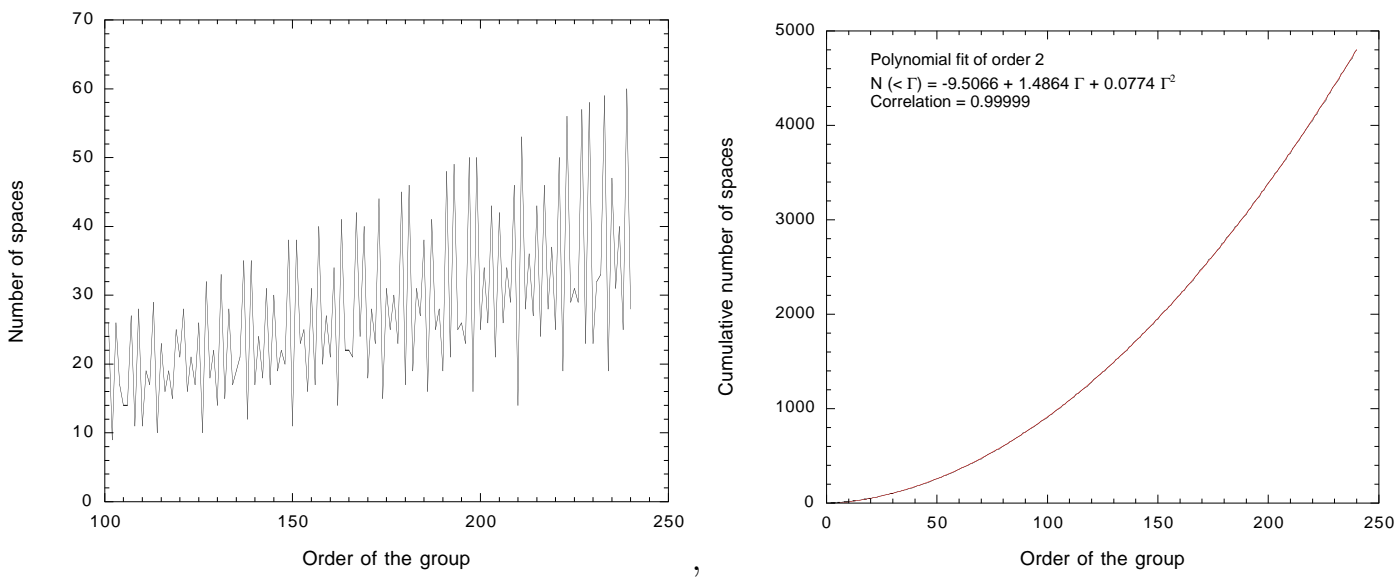

Figure 10. Left: The number of distinct spherical 3-manifolds of order exactly $|\Gamma|$. The vast majority of these manifolds are lens spaces for which there are roughly $|\Gamma|$ choices for the amount of twist in their construction. So, the number of distinct spherical manifolds of order exactly $|\Gamma|$ grows linearly with $|\Gamma|$. Right: The total number of spherical 3 -manifolds of order $|\Gamma|$ or less is the sum of an arithmetic series, and therefore grows quadratically with $|\Gamma|$.

than the effective cutoff radius $\chi_{\max }$. For example, a cyclic group $Z_{n}$ will satisfy this criterion if and only if

$$
\frac{2 \pi}{n}<\chi_{\max }
$$

Figure 12 shows the implications of this equation. In principle one could detect topology even if the shortest translations were almost as large as the diameter of the observable universe, i.e. $2 \chi_{\max }$, but when using statistical methods the signal would become too weak.

\subsection{Geometrical expectations}

In this section we determine which topologies are likely to be detectable for plausible values of $\chi_{\max }$ (see also [34] for detectability of lens spaces).

In a single action manifold every holonomy is a Clifford transformation, so in principle the PSH detects the entire holonomy group. In practice we see only a small portion of the covering 3-sphere. For example, with $\Omega_{m_{0}}=0.35, \Omega_{\Lambda_{0}}=0.75$, and a redshift cutoff of $z=3$, we see a ball of radius $\chi_{\max } \simeq 1 / 2$ (see figure 11 ). With this horizon size the binary tetrahedral, binary octahedral, and binary icosahedral groups would be hard to detect because their shortest translations distances are $2 \pi / 6$, $2 \pi / 8$, and $2 \pi / 10$, respectively. If, however, we extend the redshift cutoff to $z=1000$, then the horizon radius expands to $\chi \simeq 1$, putting the binary tetrahedral, binary octahedral, and binary icosahedral groups within the range of CMB methods.

The cyclic groups $Z_{n}$ and the binary dihedral groups $D_{m}^{*}$ are much more amenable to detection, because the shortest translation distances, $2 \pi / n$ and $2 \pi / 2 m$ respectively, can be arbitrarily small for sufficiently large $n$ and $m$. For the example given above with $\chi_{\max } \simeq 1 / 2$, a cyclic group $Z_{n}$ will be detectable if $n>2 \pi /(1 / 2) \simeq 12$, and similarly a binary dihedral group will be detectable if $2 m>12$. On the other hand, 


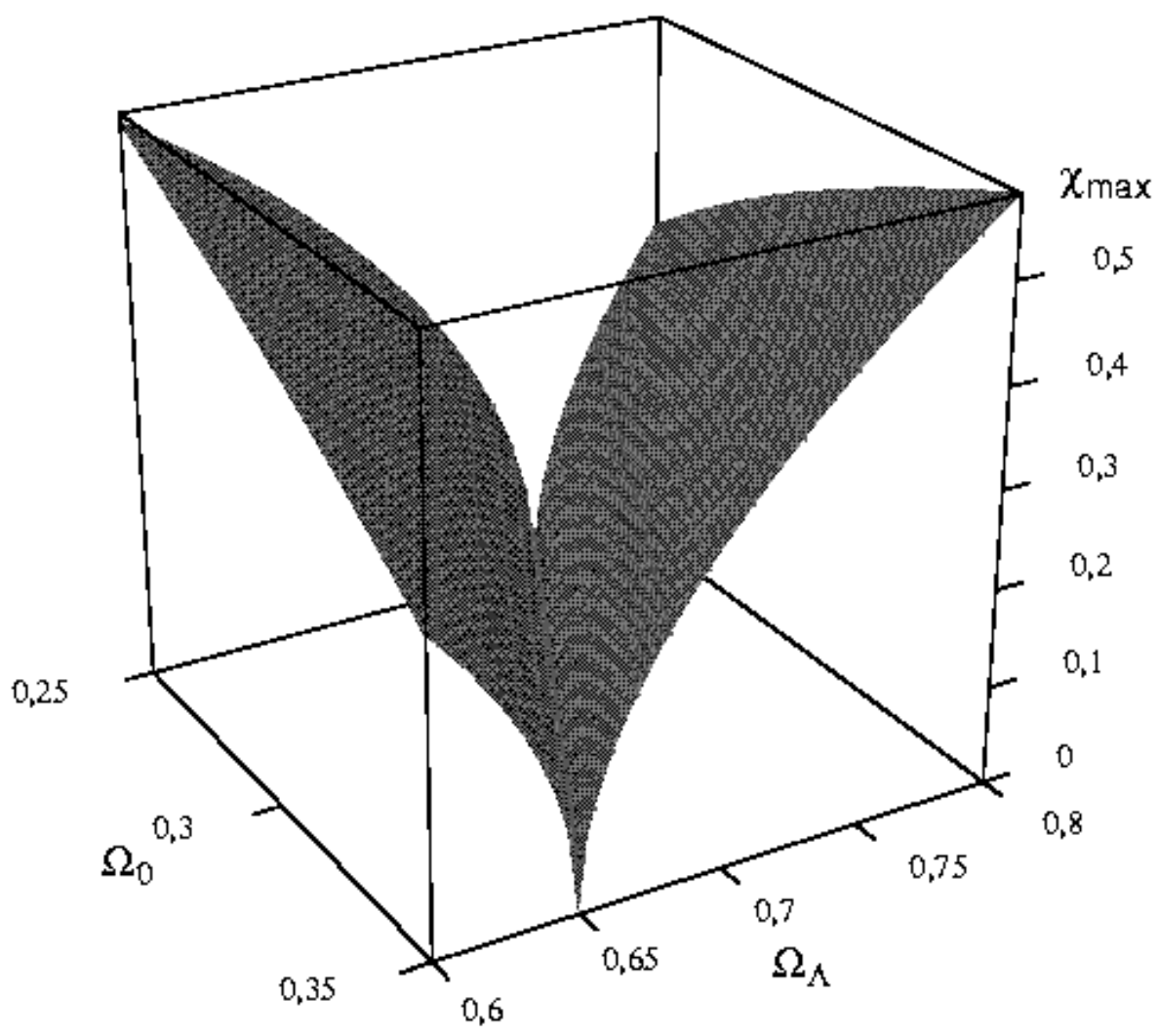

Figure 11. The maximum radial distance $\chi_{\max }$ accessible in a catalog of depth $z_{c}=5$ as a function of the cosmological parameters.

the lack of obvious periodicity on a scale of $1 / 20$ the horizon radius $\S$ implies that $n$ and $2 m$ may not exceed $2 \pi /(1 / 20) \simeq 120$. In conclusion, the orders of the cyclic groups $Z_{n}$ and the binary dihedral groups $D_{m}^{*}$ that are likely to be detectable must lie in the ranges

$$
12 \lesssim n \lesssim 120 \text { and } 12 \lesssim 2 m \lesssim 120
$$

The holonomy group of a double action manifold (recall $\S 4.2$ ) consists of the products $r l$, as $r$ ranges over a group $R$ of right-handed Clifford translations and $l$ ranges over a group $L$ of left-handed Clifford translations. Because only pure Clifford translations generate spikes, the PSH detects a product $r l$ if and only if $r= \pm \mathbf{1}$ or $l= \pm \mathbf{1}$. Thus the set of spikes in the PSH for the double action manifold defined by $R$ and $L$ will be the union of the spikes for the two single action manifolds defined by $R$ and $L$ alone, or possibly $R \times\{ \pm \mathbf{1}\}$ or $L \times\{ \pm \mathbf{1}\}$. For example, if $R=D_{2}^{*}$ and $L=Z_{3}$, then we get the union of the spikes for $D_{2}^{*}$ with the spikes for $Z_{3} \times\{ \pm \mathbf{1}\}=Z_{6}$ (see figure 14). The conditions for observability thus reduce to the conditions described for a single action manifold. That is, a double action manifold is most easily detectable if one of its factors (possibly extended by $\mathbf{- 1}$ ) is a cyclic group $Z_{n}$ or a binary dihedral group 


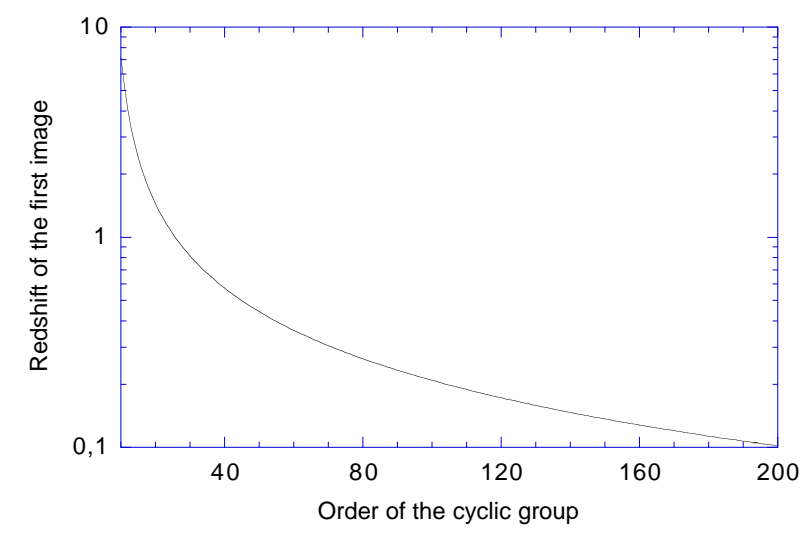

Figure 12. The redshift of the first topological image as a function of the order of the cyclic group $Z_{n}$ when $\Omega_{0}=0.35$ and $\Omega_{\Lambda_{0}}=0.75$.

$D_{m}^{*}$, with

$$
12 \lesssim n \lesssim 120 \text { and } 12 \lesssim 2 m \lesssim 120
$$

Happily the shortest translations are all Clifford translations.

In a linked action manifold (see $\S 4.3$ ) not every element of $R$ occurs with every element of $L$. Let $R^{\prime}$ be the subgroup consisting of those elements $r \in R$ that occur with $\pm \mathbf{1}$ of $L$, and let $L^{\prime}$ be the subgroup consisting of those elements $l \in L$ that occur with $\pm \mathbf{1}$ of $R$. With this notation, the Clifford translations are the union of $R^{\prime}$ and $L^{\prime}$, possibly extended by $\mathbf{- 1}$.

Linked action manifolds are the most difficult to detect observationally, because the groups $R^{\prime}$ and $L^{\prime}$ may be much smaller than $R$ and $L$. Indeed for some linked action manifolds the groups $R^{\prime}$ and $L^{\prime}$ are trivial. For example, the lens space $L(5,2)$ is obtained as follows. Let $R=Z_{5}$ and $L=Z_{5}$, and let the preferred generator $r$ of $R$ be a right-handed Clifford translation through an angle $2 \pi / 5$ while the preferred generator $l$ of $L$ is a left-handed Clifford translation through an angle $4 \pi / 5$. Link the generator $r$ to the generator $l$, and link their powers accordingly. The identity element of $R$ (resp. $L$ ) occurs only with the identity element of $L$ (resp. $R$ ), so both $R^{\prime}$ and $L^{\prime}$ are trivial. In this case the PSH contains no spikes and we must use the CCP method or CMB methods instead.

We emphasize that the detectability of a given topology depends not on the order of the group, but on the order of the cyclic factor(s). Given that we can see to a distance of about $2 \pi / 12$ in comoving coordinates (see discussion above), the lens space $L(17,1)$, a single action manifold of order 17 , would be detectable. However, the lens space $L(99,10)$, a double action manifold of order 99 generated by $Z_{9} \times Z_{11}$, would probably not be detectable, because its shortest Clifford translations have distance $2 \pi / 11$. The only way we might detect $L(99,10)$ would be if the Milky Way, just by chance, happened to lie on a screw axis, where the flow lines of the rightand left-handed factors coincide. On the other hand, a double action manifold like $S^{3} /\left(Z_{97} \times Z_{98}\right)$ probably is detectable. It has Clifford translations of order 97 and 98 , so crystallographic methods should in principle work, and it does not violate the observed lack of periodicity at $1 / 20$ the horizon scale because its screw axis (along which the minimum translation distance is $2 \pi /(97 * 98)=2 \pi / 9506)$ probably comes 
nowhere near the Milky Way.

Putting the results all together and using the complete classification of spherical spaces, we estimate that a few thousands of potentially observable topologies are consistent with current data. This number is reasonably low in view of search strategies for the detection of the shape of space through crystallogaphic or CMB methods. We note that similar lower bounds were recently obtained in [34] in the particular case of lens spaces, and this analysis does not give upper bounds and is less general than the one presented here.

\subsection{PSH spectra}

Let us now discuss the form of ideal PSH spectra. We start by considering the expected PSH spectrum in a simply-connected spherical space. As shown in [39], it is obtained by computing the probablility $\mathcal{P}\left(\chi_{a}, \chi_{l}\right) \mathrm{d} \chi_{l}$ that two points in a ball of radius $\chi_{a}$ are separated by a distance between $\chi_{l}$ and $\chi_{l}+\mathrm{d} \chi_{l}$

$$
\begin{aligned}
\mathcal{P}\left(\chi_{a}, \chi_{l}\right)= & \frac{8 \sin ^{2} \chi_{l}}{\left[2 \chi_{a}-\sin 2 \chi_{a}\right]^{2}}\left\{\left[2 \chi_{a}-\sin 2 \chi_{a}-\pi\right]+\right. \\
& \Theta\left(2 \pi-2 \chi_{a}-\chi_{l}\right) \times\left[\sin 2 \chi_{a}+\pi-\chi_{a}-\chi_{l} / 2\right. \\
& \left.\left.-\cos \chi_{a} \sec \left(\chi_{l} / 2\right) \sin \left(\chi_{a}-\chi_{l} / 2\right)\right]\right\},
\end{aligned}
$$

valid for all $\chi_{a} \in[0, \pi]$ and $\chi_{l} \in\left[0, \min \left(2 \chi_{a}, \pi\right)\right]$ and in which $\Theta$ stands for the Heavyside function.

As seen in the sample PSH spectra of figure 14, the PSH of the simply-connected space $S^{3}$ provides the background contribution of the Poisson distribution over which the spikes of the multi-connected space will appear.

Each Clifford translation $g$ of the holonomy group will generate a spike in the PSH spectrum. A Clifford translation takes the form $M(\theta, \theta)$ or $M(\theta,-\theta)$ (see $\S 3.1$ ) and so its corresonding spike occurs at $\chi=\theta$. The number of group elements sharing the same value of $|\theta|$, with $\theta$ normalized to the inverval $[-\pi, \pi]$, is the multiplicity mult $(\theta)$ of the translation distance $\theta$.

To compute the amplitude of the spike, we first consider the case that there is only one source in the fundamental polyhedron. In that case, the number of images in the covering 3-sphere equals the order of the holonomy group $|\Gamma|$. Each single image sees $\operatorname{mult}(\theta)$ neighbours lying at distance $\theta$ from it, so that this distance appears $|\Gamma| \operatorname{mult}(\theta) / 2$ times (the division by two compensates for the fact that we counted the distance from image $\mathrm{A}$ to image $\mathrm{B}$ as well as the distance from image $\mathrm{B}$ to image $\mathrm{A}$ ). We conclude that if we have $N$ sources in the fundamental polyhedron, the amplitude of the spike located at $\chi=\theta$ is

$$
\operatorname{amplitude}(\theta)=\frac{1}{2} N|\Gamma| \operatorname{mult}(\theta) .
$$

As an example, let us consider the case of the cyclic group $Z_{6}$ consisting of six Clifford translations through distances $(-2 \pi / 3,-\pi / 3,0, \pi / 3,2 \pi / 3, \pi)$. These Clifford translations yield spikes of multiplicity 1 at $\chi=0$ and $\chi=\pi$, and spikes of multiplicity 2 at $\chi=\pi / 3$ and $\chi=2 \pi / 3$, as shown in table 2. Applying the above formula to the spike at $\chi=\pi / 3$, in the case of $N=300$ distinct sources in the fundamental domain, we expect the spike to reach a height $\frac{1}{2} N|\Gamma| \operatorname{mult}(\theta)=\frac{1}{2}(100)(6)(2)=1800$ above the background distribution, in agreement with computer simulations (see figure 13). 


\begin{tabular}{|c|ccc|}
\hline$\chi / \pi$ & 0 & $1 / 2$ & 1 \\
\hline multiplicity & 1 & 6 & 1 \\
\hline
\end{tabular}

Table 1. Position and the multiplicity of the spikes for the binary dihedral group $D_{2}^{*}$. Compare with figure 14 .

\begin{tabular}{|c|cccc|}
\hline$\chi / \pi$ & 0 & $1 / 3$ & $2 / 3$ & 1 \\
\hline multiplicity & 1 & 2 & 2 & 1 \\
\hline
\end{tabular}

Table 2. Position and the multiplicity of the spikes for the binary cyclic group $Z_{6}$. Compare with figure 14 .

\begin{tabular}{|c|ccccc|}
\hline$\chi / \pi$ & 0 & $1 / 3$ & $1 / 2$ & $2 / 3$ & 1 \\
\hline multiplicity & 1 & 2 & 6 & 2 & 1 \\
\hline
\end{tabular}

Table 3. Position and the multiplicity of the spikes for $D_{2}^{*} \times Z_{3}$. As expected, we find that it can be obtained from the data for the binary dihedral group $D_{2}^{*}$ (table 1) and $Z_{3} \times\{ \pm \mathbf{1}\}=Z_{6}$ (table 2). Compare with figure 14 .

\begin{tabular}{|c|ccccccccc|}
\hline$\chi / \pi$ & 0 & $1 / 5$ & $1 / 3$ & $2 / 5$ & $1 / 2$ & $3 / 5$ & $2 / 3$ & $4 / 5$ & 1 \\
\hline multiplicity & 1 & 12 & 20 & 12 & 30 & 12 & 20 & 12 & 1 \\
\hline
\end{tabular}

Table 4. Position and the multiplicity of the spikes for the binary icosahedral group $I^{*}$. Compare with figure 15 .

In tables 1 to 4 , we give the translation distances $|\theta|$ and the multiplicities mult $(\theta)$ for the binary dihedral group $D_{2}^{*}$, the cyclic group $Z_{6}$, the product $D_{2}^{*} \times Z_{3}$, and the binary icosahedral group $I^{*}$, respectively. They correspond to the PSH spectra shown in figures 14 and 16 . Note that the spectrum for $D_{2}^{*} \times Z_{3}$ (table 3 ) is obtained from those of the binary dihedral group $D_{2}^{*}$ (table 1) and $Z_{3} \times\{ \pm \mathbf{1}\}=Z_{6}$ (table 2).

\subsection{PSH numerical Simulations}

The previous section presented the theoretical expectations for PSH in multiply connected spherical spaces. Here we carry out numerical simulations confirming those expectations. Later in this section we will also take into account the approximate flatness of the observable universe, which implies that we are seeing only a small part of the covering space, and therefore can observe spikes only at small comoving distances $\chi$.

In figure 13 we draw the histogram for the lens space $L(6,1)$ and we check that our geometrical expectations about the positions and the heights of the spikes are satisfied.

In figures 14 to 16 we present various simulations. We have chosen to draw the normalised PSH in the covering space, i.e. the PSH divided by the number of pairs and the width of the bin, and to show in grey regions the part of the PSH that is observable if the redshift cut-off is respectively $z_{c}=1,3,1000$, which roughly corresponds to catalogs of galaxies, quasars and the CMB.

We start by showing in figure 14 the "additivity" of the spectrum by considering the group $D_{2}^{*} \times Z_{3}$ for which the positions of the spikes in its PSH can be found 
from the PSH of the binary dihedral group $D_{2}^{*}$ and of $Z_{3} \times\{ \pm \mathbf{1}\}=Z_{6}$. Indeed, the amplitude of the spikes is smaller since the same number of pairs has to contribute to more spikes.

We then show in figures 15 and 16 different groups of the same order, chosen to be 120. The aim here is first to show that the number of spikes is not directly related to the order of the group and that the order of the cyclic factor, if any, is more important. While increasing this order, the number of spikes grows but their amplitudes diminish. To get a physical understanding of this effect, we show in figure 17 the view for an observer inside the manifold for the binary octahedral group $O^{*}$, for the cyclic group $Z_{17}$, and then for their product.

Finally, we applied our calculations by applying the PSH to real data, namely a catalog of about 900 Abell and ACO clusters with published redshifts. The depth of the catalog is $z_{\text {cut }}=0.26$, corresponding to $730 h^{-1} \mathrm{Mpc}$ in a spherical universe (see [14] for a more detailed description of this catalog). The PSH exhibits no spike; this gives a constraint on the minimal size of spherical space, which corresponds to a maximum order of the cyclic factor of the holonomy group of about 200 .

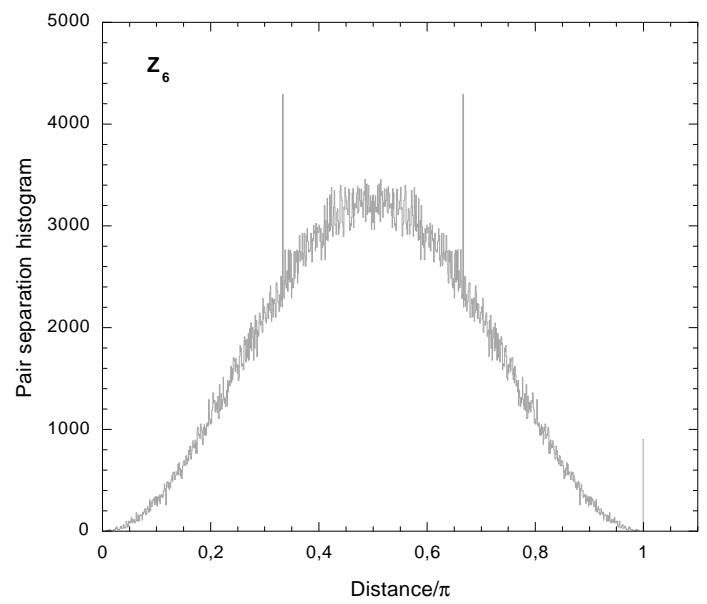

Figure 13. The PSH for the lens space $L(6,1)$ generated by the cyclic group $Z_{6}$ with $N=300$ sources in the fundamental domain. The height of the peaks at $\chi=\pi / 3$ and $\chi=2 \pi / 3$ is of order 4200 while the background distribution is of order 2400, giving the peaks a height above the background of about 1800 , in agreement with our theoretical analysis.

\subsection{Robustness of PSH}

In [20] we addressed the question of the stability of crystallograpic methods, i.e. to what extent the topological signal is robust for less than perfect data. We listed the various sources of observational uncertainties in catalogs of cosmic objects as:

(A) the errors in the positions of observed objects, namely

(A1) the uncertainty in the determination of the redshifts due to spectroscopic imprecision,

(A2) the uncertainty in the position due to peculiar velocities of objects,

(A3) the uncertainty in the cosmological parameters, which induces an error in the determination of the radial distance, 

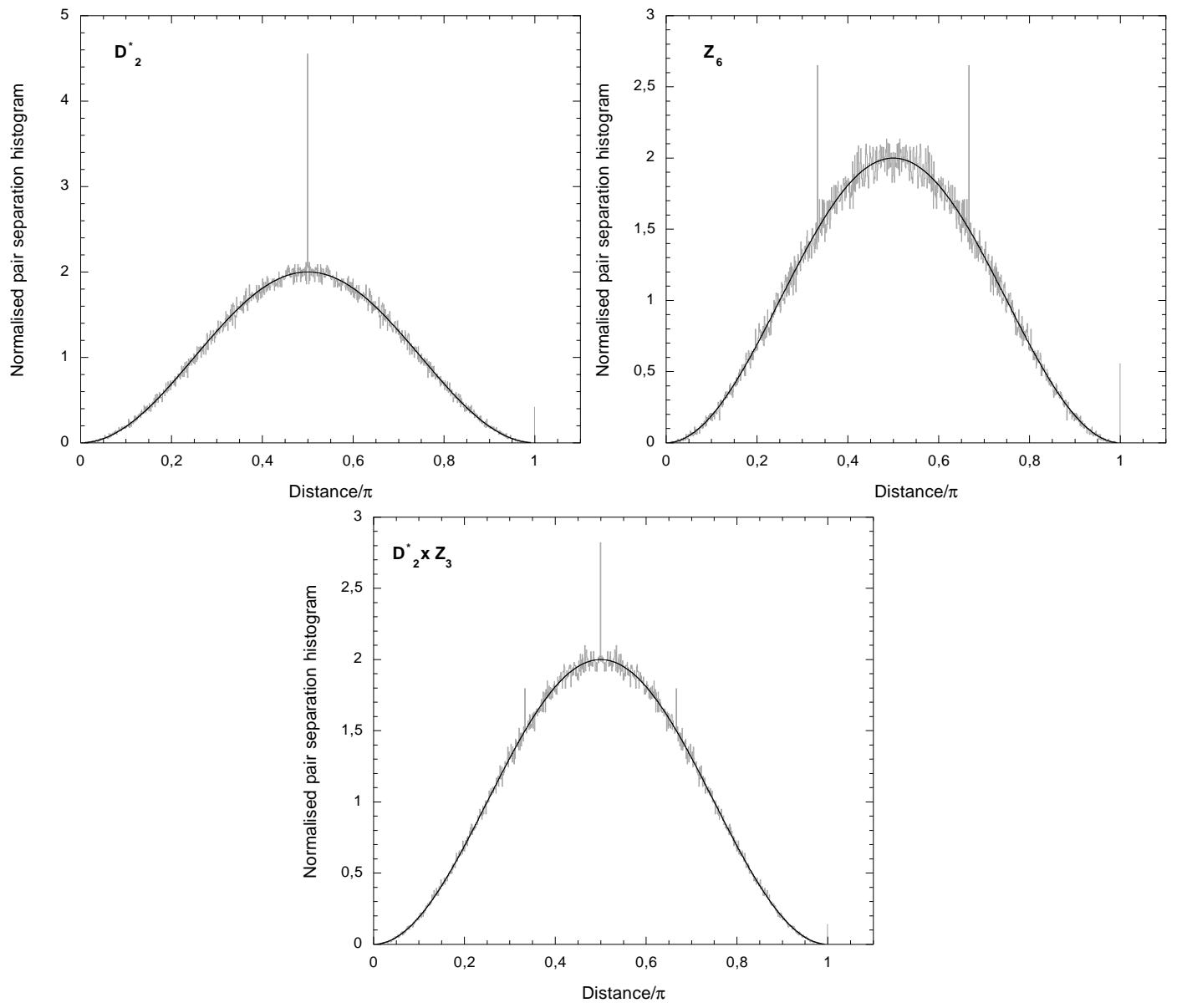

Figure 14. The PSH spectra for (upper left) the binary dihedral group $D_{2}^{*}$, (upper right) the cyclic group $Z_{6}$ and (bottom) the group $D_{2}^{*} \times Z_{3}$. One can trace the spikes from each subgroup and the black line represents the analytic distribution in a simply-connected universe.

(A4) the angular displacement due to gravitational lensing by large scale structure, (B) the incompleteness of the catalog, namely

(B1) selection effects,

(B2) the partial coverage of the celestial sphere.

We showed that the PSH method was robust to observational imprecisions, but able to detect only topologies whose holonomy groups contain Clifford translations, and we performed numerical calculations in Euclidean topologies. Fortunately the shortest translations in spherical universes are typically Clifford translations (even though more distant translations might not be), so the PSH is well suited to detecting spherical topologies. In the present work we check the robustness of PSH in spherical topologies.

Let us consider various spherical spaces of the same order 120 - namely the lens space $L(120,1)$, the binary dihedral space $D_{30}^{*}$ and the Poincaré space $I^{*}$ - and calculate the corresponding PSH's. The density parameters are fixed to $\Omega_{m_{0}}=0.35$ 

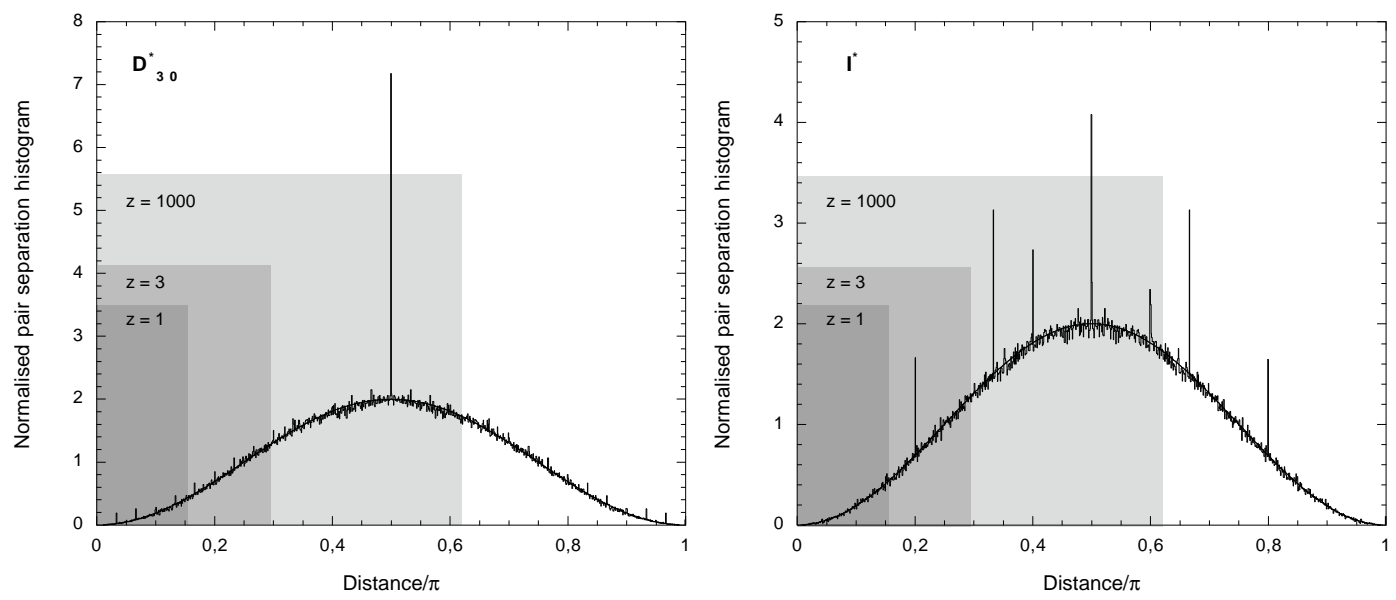

Figure 15. The PSH spectra for two spherical spaces of order 120 but with no cyclic factor: (left) the binary dihedral group $D_{30}^{*}$ and (right) the binary icosahedral group $I^{*}$

Table 5. Values of the limit percentage $p_{l}$ of rejection above which the $P S H$ spikes disappear, as a function of the redshift cut-off for the Poincaré space $I^{*}$.

\begin{tabular}{|c||c|c|c|}
\hline$z_{\text {cut }}$ & 1 & 3 & $\geq 5$ \\
\hline$p_{l}$ & No signal & $80 \%$ & $90 \%$ \\
\hline
\end{tabular}

and $\Omega_{\Lambda_{0}}=0.75$. In the runs, the number of objects in the catalog is kept constant. We examine separately the effects of errors in position due to redshift uncertainty $\Delta z$, and the effects of catalog incompleteness. Each of these effects will contribute to spoil the sharpness of the topological signal. For a given depth of the catalog, namely a redshift cut-off $z_{c u t}$, we perform the runs to look for the critical value of the error at which the topological signal fades out.

Figure 18 gives the critical redshift error $\Delta z_{l}$ above which the topological spikes disappear for the spherical space $Z_{120}$ with $\Omega_{0}=0.35, \Omega_{\Lambda 0}=0.75$.

Next, we simulate an incomplete catalog where we randomly throw out $p \%$ of the objects from the ideal catalog. For the cyclic group $Z_{120}$ and the binary dihedral group $D_{30}^{*}$, the topological signal is destroyed only for a very large rejection percentage (above $90 \%$ ). For the less favorable case of the Poincaré group $I^{*}$, the results are summarized in table 5. Thus our calculations confirm that the PSH method is perfectly robust for all spherical topologies containing Clifford translations.

As we have seen the PSH method applies for most of the spherical manifolds. Nevertheless when the order of the group or of one of its cyclic subgroups is too high then the spikes are numerous and have a small amplitude. Thus they may be very difficult to detect individually. This the case for instance for some $L(p, q)$ as well as for linked action manifolds. In that case the CCP method, which gathers the topological signal into a single index, is more suitable. Again the topological signal is rather insensitive to reasonable observational errors as soon as the underlying geometry contains enough Clifford translations. 


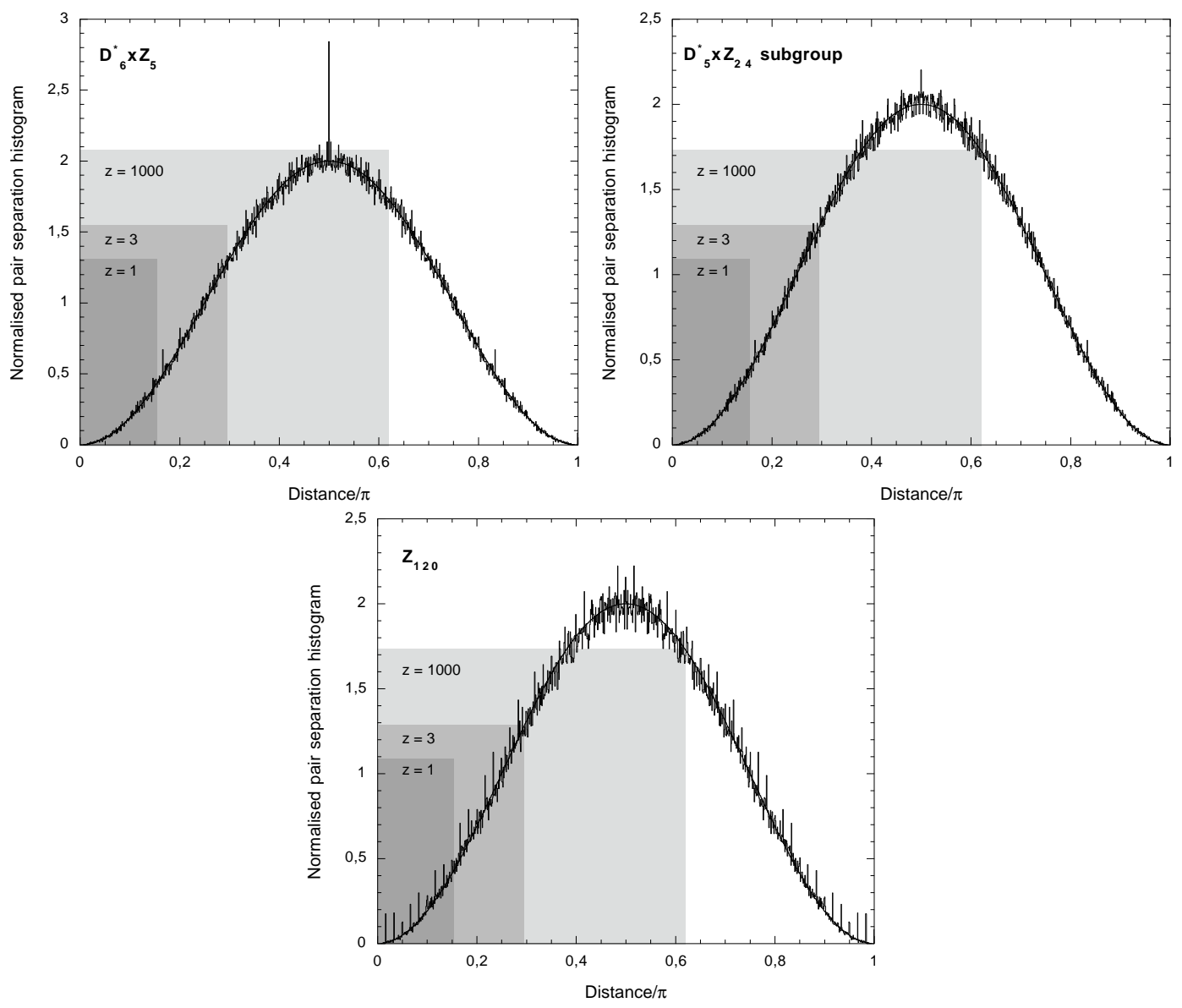

Figure 16. The PSH spectra for three spaces of order 120 with different cyclic factors: (upper left) $D_{6}^{*} \times Z_{5}^{*}$, (upper right) $D_{5}^{*} \times Z_{12}^{*}$ and (bottom) $Z_{120}$. As explained in the text, the order of the group being fixed, the higher the order of the cyclic subgroup the larger the number of spikes. However the amplitude of these spikes is smaller.

\section{Conclusion and Perspectives}

In this article, we have investigated the possible topologies of a locally spherical universe in the framework of Friedmann-Lemaitre spacetimes. We have given the first primer of the classification of three-dimensional spherical spaceforms, including the constructions of these spaces.

We have determined the topologies which are likely to be detectable in threedimensional catalogs of cosmic objects using crystallographic methods, as a function of the cosmological paramaters and the depth of the survey. The expected form of the Pair Separation Histogram is predicted, including both the background distribution and the location and height of the spikes. We have performed computer simulations of PSH in various spherical spaces to check our predictions. The stability of the method with respect to observational uncertainties in real data was also proved.

Such a complete and exhaustive investigation of the geometrical properties of 


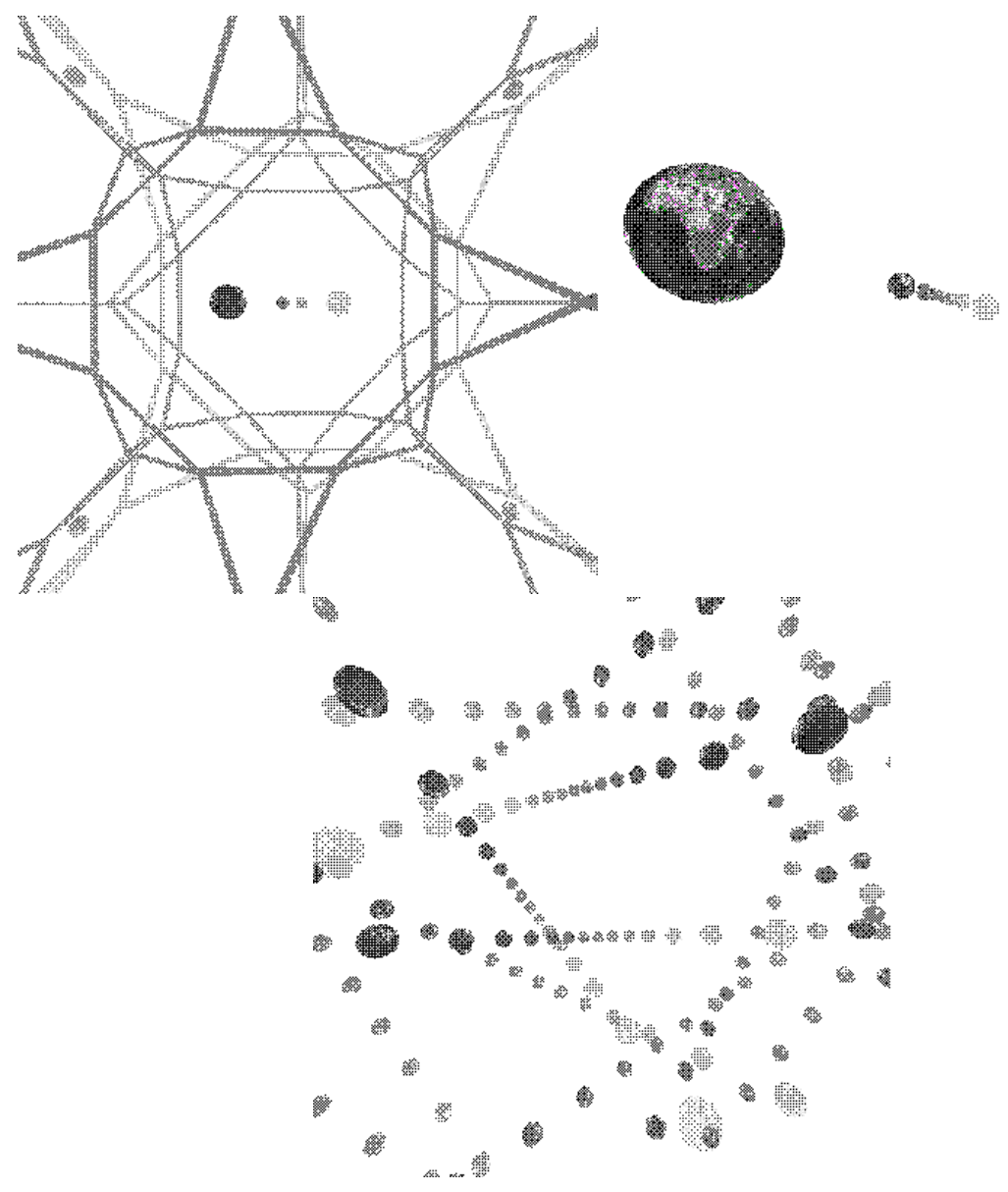

Figure 17. To have an intuitive understanding of which subgroups give rise to spikes and how, it is fruitful to have a look from the inside. In the upper left panel, we see the view inside the quotient of the 3 -sphere $S^{3}$ by the binary octahedral group $O^{*}$. The fundamental domain is a truncated cube, 48 copies of which tile $S^{3}$. The tiling's 1-dimensional edges are shown in the figure. In the upper left panel, we see the view in $L(17,1)$. All 17 translates of the Earth align along a Clifford parallel. When mixing both groups to get $O^{*} \times Z_{17}$ in the bottom panel, we see the simultaneous effects of the cyclic factor $Z_{17}$, which generates lines of images, and the binary octahedral factor, which translates one line of images to another. This illustrates how important the order of the cyclic group is, because it alone determines the distance to our nearest translate.

Note: More distant images of the Earth are always dimmer than closer images (because of artificial "fog"), but the apparent size of an image decreases only until the images reaches a distance of $\pi / 2$, after which more distant images appear larger because the light from them follows geodesics that reconverge in $S^{3}$. As an image approaches the antipodal point, at a distance of $\pi$, it fills the whole sky.

spherical spaces will be useful for further studies dealing with spherical topologies, including two-dimensional methods using $\mathrm{CMB}$ data. We plan to investigate the applicability of the circle-matching method [40] for spherical topologies. 


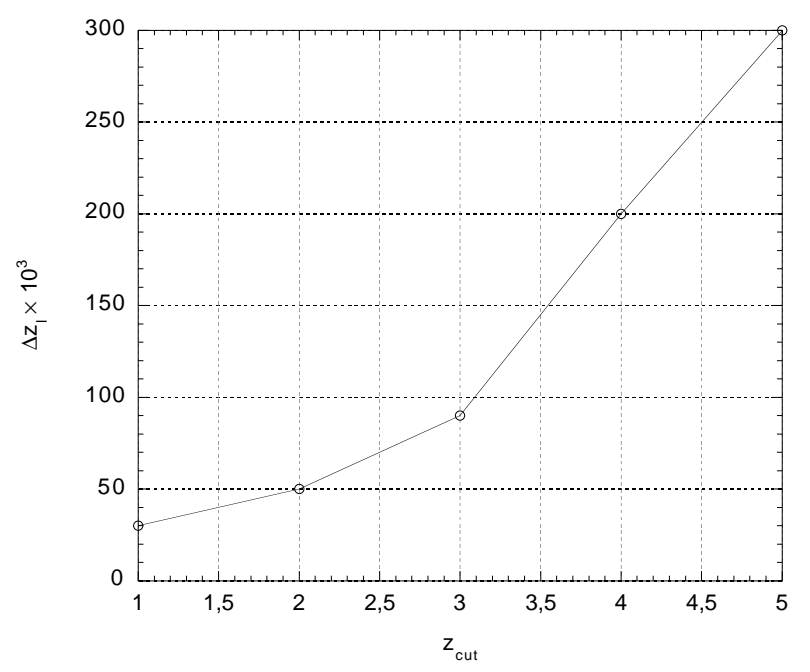

Figure 18. Plot of $\Delta z_{l}$ as a function of the depth of the catalog, for the spherical space $Z_{120}$ with $\Omega_{0}=0.35, \Omega_{\Lambda 0}=0.75$, using the PSH method.

\section{Appendix A. Quaternions}

The quaternions are a four-dimensional generalization of the familiar complex numbers. While the complex numbers have a single imaginary quantity $\mathbf{i}$ satisfying $\mathbf{i}^{2}=-1$, the quaternions have three imaginary quantities $\mathbf{i}, \mathbf{j}$, and $\mathbf{k}$ satisfying

$$
\mathbf{i}^{2}=\mathbf{j}^{2}=\mathbf{k}^{2}=-1
$$

which anti-commute

$$
\{\mathbf{i}, \mathbf{j}\}=0, \quad\{\mathbf{j}, \mathbf{k}\}=0, \quad\{\mathbf{k}, \mathbf{i}\}=0
$$

and are subject to the multiplication rules

$$
[\mathbf{i}, \mathbf{j}]=2 \mathbf{k}, \quad[\mathbf{j}, \mathbf{k}]=2 \mathbf{i}, \quad[\mathbf{k}, \mathbf{i}]=2 \mathbf{j},
$$

and

$$
[\mathbf{i}, \mathbf{1}]=0, \quad[\mathbf{j}, \mathbf{1}]=0, \quad[\mathbf{k}, \mathbf{1}]=0,
$$

where [] and \{\} are the usual commutation and anti-commutation symbols.

Geometrically, the set of all quaternions

$$
\mathbf{q}=a \mathbf{1}+b \mathbf{i}+c \mathbf{j}+d \mathbf{k}, \quad(a, b, c, d) \in R^{4}
$$

defines four-dimensional Euclidean space, and the set of all unit length quaternions, that is, all quaternions $a \mathbf{1}+b \mathbf{i}+c \mathbf{j}+d \mathbf{k}$ satisfying $a^{2}+b^{2}+c^{2}+d^{2}=1$, defines the 3 -sphere. The quaternions are associative, $(\mathbf{q r}) \mathbf{s}=\mathbf{q}(\mathbf{r s})$, even though they are not commutative. Introducing the conjugate quaternion $\overline{\mathbf{q}}$ of $\mathbf{q}$ by

$$
\overline{\mathbf{q}} \equiv a \mathbf{1}-b \mathbf{i}-c \mathbf{j}-d \mathbf{k}
$$

and the modulus of $\mathbf{q}$ by

$$
|\mathbf{q}| \equiv \sqrt{\mathbf{q} \overline{\mathbf{q}}}=\sqrt{a^{2}+b^{2}+c^{2}+d^{2}}
$$

a unit quaternion satisfies

$$
|\mathbf{q}|=1 \Longleftrightarrow \overline{\mathbf{q}}=\mathbf{q}^{-1} \text {. }
$$


The identity quaternion $\mathbf{1}$ is fundamentally different from the purely imaginary quaternions $\mathbf{i}$, $\mathbf{j}$ and $\mathbf{k}$, but among the unit length purely imaginary quaternions $b \mathbf{i}+c \mathbf{j}+d \mathbf{k}$ there is nothing special about the basis quaternions $\mathbf{i}, \mathbf{j}$ and $\mathbf{k}$. Any other orthonormal basis of purely imaginary quaternions would serve equally well.

Lemma A1: Quaternion change of basis

Let $M$ be a $3 \times 3$ orthogonal matrix, and define

$$
\begin{aligned}
\mathbf{i}^{\prime} & =M_{11} \mathbf{i}+M_{12} \mathbf{j}+M_{13} \mathbf{k} \\
\mathbf{j}^{\prime} & =M_{21} \mathbf{i}+M_{22} \mathbf{j}+M_{23} \mathbf{k} \\
\mathbf{k}^{\prime} & =M_{31} \mathbf{i}+M_{32} \mathbf{j}+M_{33} \mathbf{k}
\end{aligned}
$$

then $\mathbf{i}^{\prime}, \mathbf{j}^{\prime}$ and $\mathbf{k}^{\prime}$ satisfy the quaternion relations

$$
\begin{aligned}
& \mathbf{i}^{\prime 2}=\mathbf{j}^{\prime 2}=\mathbf{k}^{\prime 2}=-\mathbf{1} \\
& \mathbf{i}^{\prime} \mathbf{j}^{\prime}=\mathbf{k}^{\prime}=-\mathbf{j}^{\prime} \mathbf{i}^{\prime} \\
& \mathbf{j}^{\prime} \mathbf{k}^{\prime}=\mathbf{i}^{\prime}=-\mathbf{k}^{\prime} \mathbf{j}^{\prime} \\
& \mathbf{k}^{\prime} \mathbf{i}^{\prime}=\mathbf{j}^{\prime}=-\mathbf{i}^{\prime} \mathbf{k}^{\prime}
\end{aligned}
$$

Lemma A1 says that an arbitrary purely imaginary quaternion $b \mathbf{i}+c \mathbf{j}+d \mathbf{k}$ may, by change of basis, be written as $b^{\prime} \mathbf{i}^{\prime}$. If the purely imaginary quaternion $b \mathbf{i}+c \mathbf{j}+d \mathbf{k}$ has unit length, it may be written even more simply as $\mathbf{i}^{\prime}$. A not-necessarily-imaginary quaternion $a \mathbf{1}+b \mathbf{i}+c \mathbf{j}+d \mathbf{k}$ may be transformed to $a^{\prime} \mathbf{1}+b^{\prime} \mathbf{i}^{\prime}$. If it has unit length it may be written as $\cos \theta \mathbf{1}+\sin \theta \mathbf{i}^{\prime}$ for some $\theta$.

The unit length quaternions, which we continue to visualize as the 3-sphere, may act on themselves by conjugation or by left or right multiplication.

Proposition A2: Conjugation by quaternions

Let $\mathbf{q}$ be a unit length quaternion. According to the preceding discussion, we may choose a basis $\left\{\mathbf{1}, \mathbf{i}^{\prime}, \mathbf{j}^{\prime}, \mathbf{k}^{\prime}\right\}$ such that $\mathbf{q}=\cos \theta \mathbf{1}+\sin \theta \mathbf{i}^{\prime}$ for some $\theta$. It is easy to compute how $\mathbf{q}$ acts by conjugation on the basis $\left\{\mathbf{1}, \mathbf{i}^{\prime}, \mathbf{j}^{\prime}, \mathbf{k}^{\prime}\right\}$ :

$$
\begin{aligned}
& \left(\cos \theta \mathbf{1}+\sin \theta \mathbf{i}^{\prime}\right) \mathbf{1}\left(\cos \theta \mathbf{1}-\sin \theta \mathbf{i}^{\prime}\right)=\mathbf{1} \\
& \left(\cos \theta \mathbf{1}+\sin \theta \mathbf{i}^{\prime}\right) \mathbf{i}^{\prime}\left(\cos \theta \mathbf{1}-\sin \theta \mathbf{i}^{\prime}\right)=\mathbf{i}^{\prime} \\
& \left(\cos \theta \mathbf{1}+\sin \theta \mathbf{i}^{\prime}\right) \mathbf{j}^{\prime}\left(\cos \theta \mathbf{1}-\sin \theta \mathbf{i}^{\prime}\right)=\cos 2 \theta \mathbf{j}^{\prime}+\sin 2 \theta \mathbf{k}^{\prime} \\
& \left(\cos \theta \mathbf{1}+\sin \theta \mathbf{i}^{\prime}\right) \mathbf{k}^{\prime}\left(\cos \theta \mathbf{1}-\sin \theta \mathbf{i}^{\prime}\right)=-\sin 2 \theta \mathbf{j}^{\prime}+\cos 2 \theta \mathbf{k}^{\prime}
\end{aligned}
$$

Conjugation by any quaternion fixes $\mathbf{1}$ ("the north pole"), so the action is always confined to the "equatorial 2-sphere" spanned by $\left\{\mathbf{i}^{\prime}, \mathbf{j}^{\prime}, \mathbf{k}^{\prime}\right\}$. Within the equatorial 2 -sphere, conjugation by the particular quaternion $\cos \theta \mathbf{1}+\sin \theta \mathbf{i}^{\prime}$ rotates about the $\mathbf{i}^{\prime}$ axis through an angle $2 \theta$. Unlike the preceding action by conjugation, which always has fixed points, when the quaternions act by left or right multiplication they never have fixed points.

Proposition A3: Left multiplication by quaternions

Let $\mathbf{q}$ be a unit length quaternion. Choose a basis $\left\{\mathbf{1}, \mathbf{i}^{\prime}, \mathbf{j}^{\prime}, \mathbf{k}^{\prime}\right\}$ such that $\mathbf{q}=$ $\cos \theta \mathbf{1}+\sin \theta \mathbf{i}^{\prime}$ for some $\theta$. It is easy to compute how $\mathbf{q}$ acts by left multiplication on the basis $\left\{\mathbf{1}, \mathbf{i}^{\prime}, \mathbf{j}^{\prime}, \mathbf{k}^{\prime}\right\}$.

$$
\begin{array}{lr}
\left(\cos \theta \mathbf{1}+\sin \theta \mathbf{i}^{\prime}\right) \mathbf{1}= & \cos \theta \mathbf{1}+\sin \theta \mathbf{i}^{\prime} \\
\left(\cos \theta \mathbf{1}+\sin \theta \mathbf{i}^{\prime}\right) \mathbf{i}^{\prime}= & -\sin \theta \mathbf{1}+\cos \theta \mathbf{i}^{\prime} \\
\left(\cos \theta \mathbf{1}+\sin \theta \mathbf{i}^{\prime}\right) \mathbf{j}^{\prime}= & \cos \theta \mathbf{j}^{\prime}+\sin \theta \mathbf{k}^{\prime} \\
\left(\cos \theta \mathbf{1}+\sin \theta \mathbf{i}^{\prime}\right) \mathbf{k}^{\prime}= & -\sin \theta \mathbf{j}^{\prime}+\cos \theta \mathbf{k}^{\prime} .
\end{array}
$$


We see that left multiplication rotates $\mathbf{1}$ towards $\mathbf{i}^{\prime}$ while simultaneously rotating $\mathbf{j}^{\prime}$ towards $\mathbf{k}^{\prime}$. The result is a screw motion along so-called Clifford parallels. The Clifford parallels are geodesics, and they are homogeneous in the sense that there is a parallelpreserving isometry of $S^{3}$ taking any one of them to any other (see section 3.1). Action by right multiplication is similar, but yields left-handed Clifford parallels instead of right-handed ones.

\section{Appendix B. Matrices}

This appendix defines each finite subgroup of $S O(3)$ as an explicit set of rotations.

Lifting a subgroup of $S O(3)$ to the corresponding binary subgroup of $\mathcal{S}^{3}$ is easy.

Proposition A.2, together with the change of basis principle in Proposition A.1, implies that a rotation through an angle $\theta$ about an axis $(x, y, z)$ is realized by both the unit length quaternion

$$
\mathbf{q}=\cos \frac{\theta}{2} \mathbf{1}+\frac{1}{\sqrt{x^{2}+y^{2}+z^{2}}} \sin \frac{\theta}{2}(x \mathbf{i}+y \mathbf{j}+z \mathbf{k})
$$

and by its negative. This one-to-two mapping yields a binary subgroup of $\mathcal{S}^{3}$ that is exactly twice as big as the original subgroup of $S O(3)$.

Converting from a quaternion in $\mathcal{S}^{3}$ to a matrix in $S O(4)$ is also easy. When a quaternion $\mathbf{q}=a \mathbf{1}+b \mathbf{i}+c \mathbf{j}+d \mathbf{k}$ acts on the left its effect on the basis vectors $\mathbf{1}, \mathbf{i}, \mathbf{j}$, and $\mathbf{k}$ is, respectively,

$$
\begin{aligned}
& (a \mathbf{1}+b \mathbf{i}+c \mathbf{j}+d \mathbf{k}) \mathbf{1}=a \mathbf{1}+b \mathbf{i}+c \mathbf{j}+d \mathbf{k} \\
& (a \mathbf{1}+b \mathbf{i}+c \mathbf{j}+d \mathbf{k}) \mathbf{i}=-b \mathbf{1}+a \mathbf{i}+d \mathbf{j}-c \mathbf{k} \\
& (a \mathbf{1}+b \mathbf{i}+c \mathbf{j}+d \mathbf{k}) \mathbf{j}=-c \mathbf{1}-d \mathbf{i}+a \mathbf{j}+b \mathbf{k} \\
& (a \mathbf{1}+b \mathbf{i}+c \mathbf{j}+d \mathbf{k}) \mathbf{k}=-d \mathbf{1}+c \mathbf{i}-b \mathbf{j}+a \mathbf{k}
\end{aligned}
$$

Thus, relative to the orthonormal basis $\{\mathbf{1}, \mathbf{i}, \mathbf{j}, \mathbf{k}\}$, the matrix for this action is

$$
M_{\mathrm{left}}(\mathbf{q})=\left(\begin{array}{rrrr}
a & -b & -c & -d \\
b & a & -d & c \\
c & d & a & -b \\
d & -c & b & a
\end{array}\right) .
$$

Similarly, when the same quaternion $\mathbf{q}=a \mathbf{1}+b \mathbf{i}+c \mathbf{j}+d \mathbf{k}$ acts on the right, the resulting isometry has matrix

$$
M_{\text {right }}(\mathbf{q})=\left(\begin{array}{rrrr}
a & -b & -c & -d \\
b & a & d & -c \\
c & -d & a & b \\
d & c & -b & a
\end{array}\right) .
$$

In the case of double action and linked action manifolds ( $\$ 4.2$ and $\S 4.3)$, one quaternion $r$ acts on the left (giving a right-handed Clifford translation) while a different quaternion $l$ acts on the right (giving a left-handed Clifford translation). Their combined action is given by the product of their respective matrices. The order in which we multiply the two matrices doesn't matter because right- and left-handed Clifford translations always commute.

The following realizations of the finite subgroups of $S O(3)$ are unique up to an orthonormal change of coordinates. 
Appendix B.1. Cyclic Groups $Z_{n}$

Each cyclic group $Z_{n}$ consists of rotations about the axis $(0,0,1)$ through an angle $2 \pi k / n$, for $0 \leq k<n$. This defines $n$ distinct rotations.

\section{Appendix B.2. Dihedral Groups $D_{m}$}

The dihedral group $D_{m}$ contains the $m$ rotations of the cyclic group $Z_{m}$ about the axis $(0,0,1)$ as well as $m$ half turns about the axes $\left(\cos \frac{k \pi}{m}, \sin \frac{k \pi}{m}, 0\right)$ for $0 \leq k<m$. This defines $2 m$ distinct rotations.

\section{Appendix B.3. The Tetrahedral Group T}

The tetrahedral group (Figure B1) consists of

- the identity,

- order 2 rotations about the midpoints of the tetrahedron's edges, realized as $\pi$ rotations about the three axes $(1,0,0),(0,1,0)$, and $(0,0,1)$, and

- order 3 rotations about the tetrahedron's vertices and face centers, realized as counterclockwise $2 \pi / 3$ rotations about the eight axes $( \pm 1, \pm 1, \pm 1)$.

This defines a total of $1+3+8=12=|T|$ distinct rotations. Applying equation (B.1) yields the 24 quaternions of the binary tetrahedral group $T^{*}$.

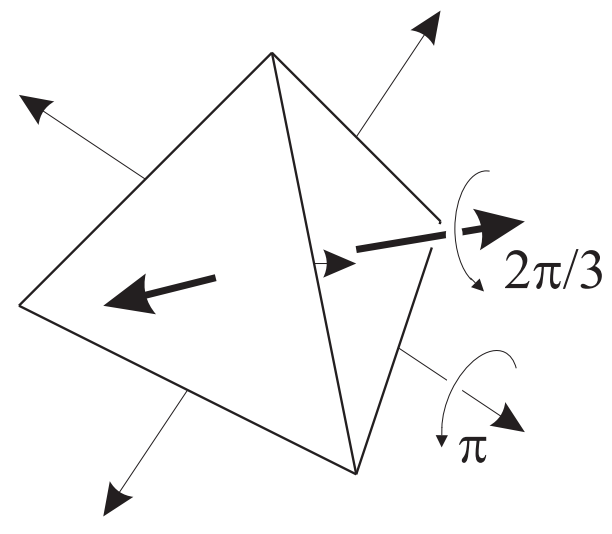

Figure B1. Visualisation of the symmetry group $T$ of the tetrahedron.

Appendix B.4. The Octahedral Group $O$

The octahedral group consists of

- the identity,

- order 2 rotations about the midpoints of the octahedron's edges, realized as $\pi$ rotations about the six axes $( \pm 1,1,0),(0, \pm 1,1)$, and $(1,0, \pm 1)$, and

- order 2 rotations about the octahedron's vertices, realized as $\pi$ rotations about the three axes $(1,0,0),(0,1,0)$, and $(0,0,1)$, and

- order 3 rotations about the centers of the octahedron's faces, realized as counterclockwise $2 \pi / 3$ rotations about the eight axes $( \pm 1, \pm 1, \pm 1)$. 
- order 4 rotations about the octahedron's vertices, realized as counterclockwise $\pi / 2$ rotations about the six axes $( \pm 1,0,0),(0, \pm 1,0)$, and $(0,0, \pm 1)$.

This defines a total of $1+6+3+8+6=24=|O|$ distinct rotations. Applying equation (B.1) yields the 48 quaternions of the binary octahedral group $O^{*}$. Note that the octahedral group contains the tetrahedral group within it.

\section{Appendix B.5. Icosahedral Group I}

The icosahedron's twelve vertices being at $( \pm \phi, \pm 1,0),(0, \pm \phi, \pm 1)$, and $( \pm 1,0, \pm \phi)$, where $\phi=(\sqrt{5}-1) / 2$ is the golden ratio, the icosahedral group consists of

- the identity,

- order 2 rotations about the midpoints of the icosahedron's edges, realized as $\pi$ rotations about the fifteen axes $(1,0,0),(0,1,0),(0,0,1),( \pm \phi, \pm(\phi+1), 1)$, $(1, \pm \phi, \pm(\phi+1))$, and $( \pm(\phi+1), 1, \pm \phi)$, and

- order 3 rotations about the centers of the icosahedron's faces, realized as counterclockwise $2 \pi / 3$ rotations about the twenty axes $( \pm 1, \pm 1, \pm 1),(0, \pm(\phi+$ $2), \pm 1),( \pm 1,0, \pm(\phi+2)),( \pm(\phi+2), \pm 1,0)$, and

- order 5 rotations about the icosahedron's vertices, realized as both counterclockwise $2 \pi / 5$ rotations and counterclockwise $4 \pi / 5$ rotations about the twelve axes $( \pm \phi, \pm 1,0),(0, \pm \phi, \pm 1)$, and $( \pm 1,0, \pm \phi)$.

This defines a total of $1+15+20+24=60=|I|$ distinct rotations. Applying equation (B.1) yields the 120 quaternions of the binary icosahedral group $I^{*}$. Note that the icosahedral group also contains the tetrahedral group within it.

\section{Acknowledgments}

JRW thanks the MacArthur Foundation for its support, and thanks the remaining authors for their warm hospitality. E.G. thanks FAPESP-Brazil (Proc. 00/04911-8) for financial support.

[1] A.G. Riess et al., Astron. J. 116 (1998) 1009; S.Perlmutter et al., Nature (London) 391 (1998) 51.

[2] P. de Bernardis et al., Nature (London) 404 (2000) 955; ibid, [astro-ph/0105296].

[3] Y. Mellier, Ann. Rev. Astron. Astrophys. 37 (1999) 127.

[4] R. Juszkiewicz et al., Science 287 (2000) 109.

[5] B. Roukema and G. Mamon, Astron. Astrophys. 358 (2000) 395.

[6] C. Wetterich, Nucl. Phys. B302 (1988) 668; R.R. Caldwell, R. Dave, and P.J. Steinhardt, Phys. Rev. Lett. 80 (1998) 1582; I. Zlatev, L. Wang, and P.J. Steinhardt, Phys. Rev. Lett. 82 (1999) 896; P.G. Ferreira and M. Joyce, Phys, Rev. D58 (1998) 023503; J.-P. Uzan, Phys. Rev. D59 (1999) 123510.

[7] M. White, D. Scott, and E. Pierpaoli, [astro-ph/0004385].

[8] A. H. Jaffe et al., Phys. Rev. Lett. 86 (2000) 3475.

[9] G.D. Mostow, Ann. Math. Studies 78 (1973), Princeton University Press, Princeton, New Jersey.

[10] M. Lachièze-Rey and J.-P. Luminet, Phys. Rep. 254 (1995) 135.

[11] J.-P. Uzan, Int. J. Theor. Physics 36 (1997) 2439.

[12] J-P. Luminet and B.F. Roukema, in Theoretical and Observational Cosmology, M. Lachièze-Rey (Ed.), Kluwer Ac. Pub., pp.117-157, [astro-ph/9901364].

[13] J-P. Uzan, R. Lehoucq, and J-P. Luminet, Proc. of the XIX ${ }^{\text {th }}$ Texas meeting, Paris 14-18 December 1998, Eds. E. Aubourg, T. Montmerle, J. Paul and P. Peter, article n ${ }^{\circ}$ 04/25, [gr-qc/0005128].

[14] R. Lehoucq, M. Lachièze-Rey, and J-P. Luminet, Astron. Astrophys. 313 (1996) 339.

[15] R. Lehoucq, J-P. Luminet, and J-P. Uzan, Astron. Astrophys. 344 (1999) 735. 
[16] G.I. Gomero, A.F.F. Texeira, M.J. Rebouças, and A. Bernui, [gr-qc/9811038].

[17] H.V. Fagundes and E. Gausmann, [astro-ph/9811368].

[18] H.V. Fagundes and E. Gausmann, Phys. Lett. A261 (1999) 235.

[19] G.I. Gomero, M.J. Rebouças, and A.F.F. Teixeira, [astro-ph/9909078]; ibid., [astro-ph/9911049].

[20] R. Lehoucq, J-P. Uzan, and J-P. Luminet, Astron. Astrophys. 363 (2000) 1.

[21] J-P. Uzan, R. Lehoucq, and J-P. Luminet, Astron. Astrophys. 351 (1999) 766.

[22] J.A. Wolf, Spaces of constant curvature, fifth edition, Publish or Perish Inc., Wilmington USA (1967).

[23] A.F. Beardon, The geometry of discrete groups, New York, Springer (1983).

[24] M. Nakahara, Geometry, Topology and Physics, Adam Hilger, Bristol, New-York (1990).

[25] W. de Sitter, Month. Not. R. Astron. Soc 78 (1917) 3 ; Proceedings of the Royal Academy of Amsterdam 20 (1917) 229.

[26] J.-P. Luminet, L'invention du big bang, introduction to Essais de Cosmologie de A. Friedmann et G. Lemaître, Seuil, Paris, 1997, p. 78.

[27] A.S. Eddington, The mathematical theory of relativity, chap. 5, (Cambridge University Press, Cambridge, 1923).

[28] A. Friedmann, Zeitschr. für Phys. 2 (1924) 326.

[29] G. Lemaître, Rev. Quest. Sci. (1929) 189 (English translation in The Primeval atom, Van Nostrand, New York, 1950).

[30] J.V. Narlikar and T.R. Seshadri, Astrophys. J. 288 (1985) 43.

[31] G.F. R. Ellis, Gen. Rel. Grav. 2 (1971) 7.

[32] J. R. Gott, Month. Not. R. Astron. Soc. 193 (1980) 153.

[33] R. Ionicioiu and R. Williams, Class. Quant. Grav. 15 (1998) 3469.

[34] G.I. Gomero, M.J. Rebouças, and R. Tavakol, [gr-qc/0105002].

[35] W. Threlfall and H. Seifert, "Topologische Untersuchung der Diskontinuitätsbereiche endlicher Bewegungsgruppen des dreidimensionalen sphärischen Raumes", Math. Annalen 104 (1930) 1-70 and 107 (1932) 543-586.

[36] W.P. Thurston, Three-dimensional geometry and topology (1997) Princeton Mathematical series 35, Ed. S. Levy, (Princeton University Press, Princeton, USA).

[37] H. Poincaré, "Cinquième complément à l'analysis situs", Rend. Circ. Mat. Palermo 18 (1904) 45-110.

[38] C. Weber and H. Seifert, "Die beiden Dodekaederräume", Math. Zeitschrift 37 (1933) 237-253.

[39] A. Bernui and A.F.F. Teixeira, [astro-ph/9904180].

[40] N. Cornish, D. Spergel, and G. Starkman, Class. Quant. Grav. 15 (1998) 2657. 